NBER WORKING PAPER SERIES

\title{
HETEROGENEITY IN TARGET-DATE FUNDS: OPTIMAL RISK-TAKING OR RISK MATCHING?
}

\author{
Pierluigi Balduzzi \\ Jonathan Reuter \\ Working Paper 17886 \\ http://www.nber.org/papers/w17886
NATIONAL BUREAU OF ECONOMIC RESEARCH
1050 Massachusetts Avenue
Cambridge, MA 02138
March 2012

Both authors are from Boston College. The authors thank Ryan Alfred and Brooks Herman of BrightScope for providing them with retirement plan-level data, and Lauren Beaudette and Bianca Werner for excellent research assistance. The authors also thank John Beshears (discussant), Jeffrey Brown, Bjarne Astrup Jensen (discussant), Stephen Utkus (dicussant), Mark Warshawsky (discussant), and seminar participants at Boston College, the 13th Annual Retirement Research Consortium Conference, the 2012 European Finance Association meetings, and the 2015 Wharton Conference on Financial Decisions and Asset Markets. Corresponding author: Jonathan Reuter, Carroll School of Management, Boston College, 140 Commonwealth Avenue, Chestnut Hill, Massachusetts, 02467; Tel: (617) 552-2863; Fax: (617) 552-0431; email: reuterj@bc.edu. The research was supported by a grant from the U.S. Social Security Administration (SSA) as part of the Retirement Research Consortium (RRC). The findings and conclusions expressed are solely those of the authors and do not represent the views of SSA, any agency of the federal government, Boston College, or the National Bureau of Economic Research. An earlier version of this paper was titled "Heterogeneity in Target-Date Funds and the Pension Protection Act of 2006."

NBER working papers are circulated for discussion and comment purposes. They have not been peerreviewed or been subject to the review by the NBER Board of Directors that accompanies official NBER publications.

(C) 2012 by Pierluigi Balduzzi and Jonathan Reuter. All rights reserved. Short sections of text, not to exceed two paragraphs, may be quoted without explicit permission provided that full credit, including (C) notice, is given to the source. 
Heterogeneity in Target-Date Funds: Optimal Risk-Taking or Risk Matching?

Pierluigi Balduzzi and Jonathan Reuter

NBER Working Paper No. 17886

March 2012, Revised June 2013, Revised July 2015

JEL No. G11,G18,G23

\begin{abstract}
$\underline{\text { ABSTRACT }}$
Following the Pension Protection Act of 2006, there was a sharp increase in the use of TDFs as default investment options in defined contribution retirement plans. We document large differences in realized TDF returns and risk profiles, even for funds with the same target retirement date. Using fund-level data, we find evidence that this heterogeneity reflects optimal risk-taking by fund families with low market share, especially those entering the market after 2006. Using plan-level data, we find little evidence that $401(\mathrm{k})$ plan sponsors match the risk profile of the TDFs in their plans to the risks of their companies.
\end{abstract}

\author{
Pierluigi Balduzzi \\ Carroll School of Management \\ Boston College \\ 330B Fulton Hall \\ 140 Commonwealth Avenue \\ Chestnut Hill, MA 02467 \\ pierluigi.balduzzi@bc.edu \\ Jonathan Reuter \\ Carroll School of Management \\ Boston College \\ 224B Fulton Hall \\ 140 Commonwealth Avenue \\ Chestnut Hill, MA 02467 \\ and NBER \\ reuterj@bc.edu
}




\section{Introduction}

A common implication of normative optimal portfolio models is that, as investors age, it is optimal for them to shift their financial wealth away from stocks and toward bonds. ${ }^{1}$ This normative implication has found its way into the design of investment products: target-date mutual funds (TDFs). ${ }^{2}$ Wells Fargo introduced the first TDFs in 1994. According to Seth Harris, Deputy Secretary of the Department of Labor (DOL), TDFs "were designed to be simple, long-term investment vehicles for individuals with a specific retirement date in mind." ${ }^{3}$ Investors who plan to retire in 2030, for example, are encouraged to invest all of their 401(k) assets in the Wells Fargo LifePath 2030 fund. The innovation, relative to traditional balanced funds (BFs), is that TDFs relieve investors of the need to make asset allocation decisions: when the target date is far away, the TDF invests primarily in risky assets, such as domestic and foreign equity and, as the number of years to the target date declines, the TDF automatically reduces its exposure to risk. The formula used to determine how a TDF's asset allocation changes as the number of years to the target date declines is known as the "glide path." The promise of a simple, long-term retirement investment prompted the DOL, through the Pension Protection Act of 2006 (PPA), to allow firms to adopt TDFs as default investment vehicles in employer-sponsored defined contribution (DC) retirement plans.

More recently, however, policy makers have begun to worry about risk-taking by TDFs. In 2009, Herb Kohl, chairman of the Senate Special Committee on Aging, wrote: "While well-

\footnotetext{
${ }^{1}$ Merton (1971) shows that when an investor faces time-series variation in the first and second conditional moments of asset returns, her optimal portfolio is composed of both a myopic component and an intertemporal component, the "hedging" demand. Balduzzi and Lynch (1999) and Lynch (2001) argue that mean reversion in equity prices causes the hedging demand for equity to decrease as the investment horizon decreases. Jagannathan and Kocherlachota (1996) and Cocco et al. (2005) argue that older workers should allocate more of their financial wealth to bonds, because they can expect to receive shorter streams of bond-like income from their human capital. Bodie et al. (1992) come to the same conclusion by arguing that older workers have fewer opportunities to adjust their labor supply in response to realized returns on their assets. One qualification comes from Benzoni et al. (2007), who note that when labor income and dividends are co-integrated, the demand for equity over the life cycle should be hump-shaped, rather than monotonically decreasing. Another qualification comes from Pástor and Stambaugh (2011), who argue that in the presence of parameter uncertainty and imperfect predictability, the optimal equity allocation should depend not only on the remaining time until retirement, but also on the initial length of the investor's horizon.

${ }^{2}$ TDFs are also referred to as lifecycle funds.

${ }^{3}$ DOL and SEC Joint Public Hearing on TDFs and Other Similar Investment Options: June 18, 2009.
} 
constructed target date funds have great potential for improving retirement income security, it is currently unclear whether investment firms are prudently designing these funds in the best interest of the plan sponsors and their participants. In fact, an Aging Committee investigation conducted in early 2009 found significant differences in the asset allocations and equity holdings within these funds, raising questions about whether plan sponsors and participants understand the underlying assumptions and risk associated with these products" (Special Committee on Aging (2009)). ${ }^{4}$

In summary, there are at least two reasons why it is important to study the market for TDFs. First, this is a relatively new market whose size has increased markedly since the passage of the PPA of 2006. Hence, the TDF market is a "laboratory" in which we can study how mutual fund families structure new investment products and compete for market share. Second, TDFs are quickly becoming the default investment option of choice in DC retirement plans. Hence, given the increasing role of defined contribution plans in the funding of retirement, the investment behavior of TDFs has special policy significance.

We hypothesize two reasons why realized returns and risk characteristics may differ markedly across TDFs with the same target date. First, mutual fund families may decide to increase their risk exposure as a way to increase market share. This is the "optimal risk-taking" hypothesis. Second, families may exploit clientele effects in the defined contribution (DC) plan market. Namely, families may offer TDFs with different risk profiles so that plan sponsors can choose family whose TDFs best offset the risk from being employed in a given firm or industry, or match the overall risk preferences of the employees covered by their DC plans. This is the "risk-matching" hypothesis.

Characterizing the heterogeneity in realized TDF returns and the risk characteristics of returns is important because TDF investors may have thought that two TDFs with the same target date are close substitutes. We show that this is not necessarily the case. This matters especially

\footnotetext{
${ }^{4}$ As highlighted in Tables 2-6, the heterogeneity in various aspects of TDF returns became especially apparent during 2008-2009. In other words, the financial crisis amplified differences in TDF return properties that, as argued in this paper, were mainly brought about by the entrance of new families after the PPA.
} 
for investors who access TDFs through their employer-sponsored defined contribution retirement plan and are typically limited to the TDFs offered by a single fund family. ${ }^{5}$ Understanding the economic determinants of the heterogeneity in returns is important because, if the heterogeneity is driven by fund families responding to risk-taking incentives, then it is likely that this is "bad" heterogeneity from the investor's perspective. Alternatively, if the heterogeneity is driven by risk matching, then it may reflect the attempt on the part of plan sponsors to compensate for the high or low risk of employees' human capital, or to match employees' risk preferences with the risk of the TDF, which we would classify as "good" heterogeneity.

We begin our empirical analysis by documenting that the dispersion in realized TDF returns between 2000 and 2012 is substantial, especially when we focus on the years immediately after the PPA. For example, in 2009, there are 67 TDFs with target dates of 2015 or 2020 . The average annual realized return in this sample of TDFs is $25.1 \%$, the cross-sectional standard deviation is $4.4 \%$, and the range (the difference between the maximum and minimum return) is $23.5 \%$. Importantly, a similar pattern holds for the idiosyncratic component of realized returns, "alpha." In 2009, the cross-sectional standard deviation of alphas from a five-factor model for these TDFs is $3.1 \%$ and the range is $12.9 \%$. While the $R^{2}$ s of a five-factor model average $97.3 \%$ for $2015-2020$ TDFs in 2009 , the minimum is $84.8 \%$, indicating substantial differences in systematic risk as well. Indeed, within the sample of 2015-2020 TDFs in 2009, the standard deviation of the beta on U.S. equity is 0.12 , and the range is 0.64 .

In summary, the substantial heterogeneity in realized TDF returns reflects heterogeneity in both idiosyncratic and systematic risk. If regulators had assumed that TDFs with the same target date would provide investors with the same exposure to risk, they were mistaken. While different

\footnotetext{
${ }^{5}$ Among the 8,406 plans in our BrightScope data that offer TDF mutual funds, 95.9\% offer TDFs from a single mutual fund family, 3.4\% offer TDFs from two families, and $0.7 \%$ offer TDFs from three or more families. Larger plans are slightly more likely to offer TDFs from multiple families because they are more likely to use multiple record keepers: the correlation between plan assets and the number of families offering TDFs is 0.15.
} 
investors may prefer TDFs with different risk exposures, risk matching requires that investors (and plan sponsors) are able to evaluate the level of risk. To the extent that a large portion of TDF risk is idiosyncratic risk, the glide path becomes uninformative.

To rationalize the increased dispersion in idiosyncratic risk, we consider three incentives related to risk-taking. First, given that TDF flows are likely driven by the choice of plan sponsors, TDF flows are likely to respond more strongly to risk-adjusted performance than the typical retail fund. Second, by increasing the expected market share of TDFs inside retirement plans, the PPA increased the incentive for fund families to enter this market. Between 2006 and 2012, assets under management in TDFs more than quadrupled, increasing from $\$ 116.0$ billion to $\$ 480.2$ billion, and the number of mutual fund families offering TDFs jumped from 27 to 44, before falling back down to 37. Third, the fact that that new entrants have few assets under management to lose adds convexity in the flow-performance relation and, therefore, an additional incentive to engage in risktaking. ${ }^{6}$ These three incentives lead us to predict that the heterogeneity discussed above is mainly driven by post-PPA entrants with low market share.

Our findings are broadly consistent with optimal risk-taking. First, we document that flows into TDFs respond significantly to idiosyncratic returns, but not to total returns, a pattern which is consistent with plan sponsors using risk-adjusted returns to evaluate TDFs. Second, we find that families that enter the market for TDFs after 2006 and have low TDF market share, offer TDFs whose returns differ markedly from their peers. The monthly returns on these new funds differ from the average monthly return of other funds with the same target date by 90 basis pointsapproximately $11 \%$ annually. Interestingly, realized alphas for these new TDFs differ from the average alpha by substantial amounts, as much 56 basis points. We also find that entrants tend to have more idiosyncratic risk as a fraction of total risk (as measured by the $R^{2}$ of a one- or five-factor

\footnotetext{
${ }^{6}$ The risk-taking incentives faced by a new TDF are akin to the incentives faced by new funds being "incubated" by mutual fund families; see Evans (2010).
} 
model) and more dispersion in the sensitivities of their returns to global bond and stock indices and to commodities. It is worth noting that all of our tests control for the average level of risk-taking by BFs in the same family, and that we find strongest correlations in TDF and BF behavior when we focus on the level of systematic risk (i.e., the sensitivities to the different sector indices).

An alternative explanation for the observed heterogeneity in TDFs is the "risk-matching" hypothesis: TDFs offer different levels of risk to cater to the heterogeneous needs and preferences of different investor clienteles. ${ }^{7}$ To investigate this hypothesis, we exploit newly available data from BrightScope on the investment menus of several thousand DC retirement plans in 2010. Note that this snapshot of the DC retirement plan universe takes place after the PPA of 2006 and, hence, at a time when plan sponsors have a large set of TDFs from which to choose. In other words, the passage of the PPA should make it easier to detect possible risk-matching, as well as risk-taking, effects. For firms with publicly-traded equity, we regress the systematic (idiosyncratic) risk of the 2020 TDF offered in each plan on the systematic (idiosyncratic) risk of the firm's equity. To expand our sample, we also regress the risk of the 2020 TDF offered in each plan on the median risk of firms within the same industry. Regardless of whether we focus on systematic or idiosyncratic risk, we find little evidence of risk matching. Moreover, when we include industry fixed effects to control for differences in volatility of employment and other time-invariant differences across industries, the $R^{2}$ of our regressions remain low. We conclude that riskier firms are no more or less likely to choose riskier TDFs than safer firms.

In summary, we document pronounced heterogeneity in investor exposure to both systematic and idiosyncratic risk across TDFs with the same target date. This heterogeneity increases with the passage of the PPA in 2006, which draws new families into the TDF market. We show that the decision of these families to load on idiosyncratic risk is consistent with optimal risk-

\footnotetext{
${ }^{7}$ Viceira (2009), for example, advocates that TDF glide paths be tailored to match the human capital risk and risk preferences of the employees of plan sponsor firms.
} 
taking behavior. On the other hand, we find little evidence that the heterogeneity in systematic or idiosyncratic risk-taking is driven by matching between TDF and sponsoring firm's risk characteristics. Hence, our findings support the notion that the TDF heterogeneity uncovered by this paper is bad heterogeneity, driven by risk-taking incentives, rather than good heterogeneity, driven by risk-matching motives. As we discuss, heterogeneity without risk matching can impose significant utility costs on investors.

Overall, our findings have both normative and positive relevance: From a normative standpoint, more transparency regarding TDF glide paths and systematic risk is not enough, since entrants have differentiated their products largely in terms of idiosyncratic returns. From a positive standpoint, we provide an explanation for an apparently puzzling degree of heterogeneity in realized TDF returns.

The remainder of the paper is organized as follows: Section 2 provides institutional background on the market for TDFs and a brief review of the related literature. Section 3 describes the mutual fund data used in the study. Section 4 documents cross-sectional differences in annual total and idiosyncratic returns, $R^{2} \mathrm{~s}$, and betas, for TDFs with the same target date. Section 5 tests whether the heterogeneity reflects optimal risk-taking. Section 6 describes the retirement plan-level data and tests for risk matching. Section 7 discusses the potential costs of TDF heterogeneity to investors. Section 8 concludes.

\section{Institutional background and review of the literature}

Although only four fund families offered target-date funds (TDFs) in 2000, the Pension Protection Act of 2006 (PPA) allowed firms to offer TDFs as default investment options within 401(k) retirement plans. The regulatory goal was to redirect investors from money market funds - the dominant 
default investment option - to age-appropriate, long-term investment vehicles. ${ }^{8}$ To accomplish this goal, the PPA relieves plan sponsors of liability for market losses when they default employees into a Qualified Default Investment Alternative (QDIA). The set of QDIAs is limited to TDFs, BFs, and managed accounts. While TDFs were perceived to be an important innovation in the market for retirement products, commentators have recently expressed concerns about the lack of transparency regarding risk. ${ }^{9}$

The Investment Company Institute (ICI) reports that the share of 401(k) plans offering TDFs increased from $57 \%$ in 2006 to $72 \%$ in $2012 .{ }^{10}$ Similarly, the share of $401(\mathrm{k})$ plan participants offered TDFs increased from $62 \%$ to $68 \%$. At year-end 2012, $41 \%$ of $401(\mathrm{k})$ participants held at least some plan assets in TDFs, up from $19 \%$ at year-end 2006. According to our sample of investment menus from BrightScope, approximately $10 \%$ of all 401(k) and 403(b) retirement plan assets in 2010 were invested in TDFs. However, ICI reports that 401(k) plan participants in their twenties collectively allocated $34.2 \%$ of their retirement assets to TDFs in 2012 . Therefore, employees just entering the labor force appear likely to finance their retirement through a combination of TDF returns and Social Security benefits. ${ }^{11}$ Because the PPA effectively directs investors toward TDFs, we believe that it is important to study the incentives and behavior of these emerging investment vehicles.

Interestingly, the two current leaders in the market for TDFs take very different approaches

\footnotetext{
${ }^{8}$ The tendency of investors to stick to their default investment allocation (i.e., inertia), has been discussed by Benartzi and Thaler (2001), Madrian and Shea (2001) and Agnew et al. (2003), among others.

${ }^{9}$ The Appendix, Sections A.1 and A.2, includes a detailed description of the PPA and a selection of quotes on the pros and cons of TDFs.

${ }^{10}$ All of the numbers in this paragraph except for our calculation using BrightScope data are taken from Figure 7.8 and Figure 7.10 of the 2014 Investment Company Institute Fact Book.

${ }^{11}$ As documented by Madrian and Shea (2001) and Agnew et al. (2003), 401(k) investors exhibit strong inertia in their asset allocations and, hence, young investors who have been defaulted in a TDF are likely to stay in that investment vehicle for a long time. Moreover, inertia is likely to be even more pronounced in the case of TDF investment, as TDFs are designed to adjust their allocations as investors age. In addition, Mitchell and Utkus (2012) show that, independently of default effects, new plan entrants adopted TDF voluntarily at an average $31 \%$ rate, during the 2003-2010 period. The appeal of TDFs as a long-run investment choice may derive from the fact that the funds' glide paths effectively amount to implicit investment advice; see Chalmers and Reuter (2014) and Mitchell and Utkus (2012).
} 
to the design of their products. Vanguard's approach is to allocate investments across five low cost index funds. Fidelity's approach, on the other hand, is to allocate investments across as many as 27 actively managed mutual funds. ${ }^{12}$ Whether one approach is better for investors than the other is an open question, but the two approaches highlight a significant source of heterogeneity in how TDFs are constructed.

This is the first paper to focus on the heterogeneity of TDFs and to study changes in the population of TDFs around the introduction of the PPA. The existing literature mainly compares TDFs to other investment vehicles and studies the factors driving individual demand for TDFs. ${ }^{13}$ The paper most closely related to our own is Sandhya (2011), who compares TDFs to BFs offered within the same mutual fund family. While Sandhya (2011) focuses on average differences in fund expenses and returns, our paper links heterogeneity in idiosyncratic risk to risk-taking incentives arising from the PPA. Also related is Elton et al. (2014), who use data on underlying mutual fund holdings to study both the level of TDF fees and how deviations from TDF glide paths effect fund-level returns. Their finding that TDFs have become increasing likely to invest in emerging markets, real estate, and commodities complements our findings related to heterogeneity in TDF betas. However, they do not ask whether risk-taking by entrants helps to explain the movement into new asset classes. Moreover, none of the existing papers explores the extent to which plan sponsors consider TDF risk when constructing their investment menus. ${ }^{14}$ Our unique plan-level data allow us to test for risk matching between firms and TDFs.

\footnotetext{
${ }^{12}$ Fidelity now also offers an index fund version of their TDFs, the "Fidelity Freedom Index Funds." These funds were introduced in 2009.

${ }^{13}$ Yamaguchi et al. (2007), Park and VanDerhei (2008), Park (2009), and Mitchell et al. (2009) study investor demand for the particular TDFs introduced into their samples of DC retirement plans. Pagliaro and Utkus (2010) and Mitchell and Utkus (2012) study the role of a 401(k) plan's architecture on TDF demand. Chalmers and Reuter (2014) argue that TDFs are cost-effective substitutes for financial advisors. Ameriks et al. (2011), Morrin et al. (2012), and Agnew et al. (2012) use survey data to identify the factors behind TDF investment.

${ }^{14}$ Shiller (2005), Gomes et al. (2008), and Viceira (2009) use simulations and calibrated lifecycle models to compare the properties of representative TDFs to those of other investment vehicles. Pang and Warshawsky (2009) study the effect of heterogeneity in glide paths on the distribution of terminal wealth.
} 


\section{Data}

We obtain data on mutual fund names, characteristics, fees, and monthly returns from the CRSP Survivor-Bias-Free U.S. Mutual Fund Database. CRSP does not distinguish TDFs from other types of mutual funds, but they are easily identified by the target retirement year in the fund name (e.g., AllianceBernstein 2030 Retirement Strategy). Through much of the paper, our unit of observation is family $i$ 's mutual fund with target date $j$ in month $t$. For example, T. Rowe Price offers twelve distinct TDFs in December 2012, with target dates of 2005, 2010, .., 2045, 2055, plus an income fund. As with other types of mutual funds, TDFs typically offer multiple share classes. To calculate a fund's size, we sum the assets under management at the beginning of month $t$ across all of its share classes. To calculate a fund's expense ratio, we weight each share class's expense ratio by its assets under management at the beginning of the month. To calculate a fund's age, we use the number of months since its oldest share class was introduced. To identify families that enter the market after December 31, 2006, we use the year when each mutual fund family offered its first TDF. Because we find that CRSP data on the holdings of equity, debt, and cash are unreliable for TDFs, we infer investment strategies from the betas estimated in factor models. ${ }^{15}$

Table 1 presents summary statistics on the evolution of the TDF market over the 1994-2012 period. Wells Fargo introduced the first TDFs in 1994. Between 1994 and 2012, the number of TDFs grew from five to 368 and the number of mutual fund families offering TDFs grew from one to 37 , with total assets under management going from $\$ 278$ million to $\$ 480$ billion, a seventeenhundred-fold increase. ${ }^{16}$ In particular, 20 families entered the market after 2006, allowing us to study differences between the TDFs of new entrants and more established mutual fund families.

\footnotetext{
${ }^{15}$ We document inconsistencies in CRSP equity holdings data in Section B of the Supplementary Appendix.

${ }^{16}$ The number of distinct TDFs cannot be directly calculated from Table 1 because some families offer multiple TDFs within a given range of target dates (e.g., Fidelity offers TDFs with target dates of 2015 and 2020) and some families offer multiple TDFs with a given target date (e.g., Fidelity now offers active and passive versions of each TDF).
} 
While Wells Fargo was the market leader until 1997, Fidelity took the lead in 1998. Fidelity's dominant position has been eroded, though, dropping from a maximum market share of $88.1 \%$ in 2002, to $32.7 \%$ in 2012. Similarly, although the market for TDFs remains quite concentrated, the market share of the top three firms has fallen gradually from $97.8 \%$ in 2002 , to $75.1 \%$ in 2012 . Firms that entered the market after 2006 (and remained in the market through 2012) have a combined market share of $4.4 \%$. It is worth noting that seven of the ten families that exit the TDF market between 2009 and 2012 also entered the market after 2006. These include Goldman Sachs and Oppenheimer.

We also use the CRSP mutual fund database to construct a sample of traditional (non-TDF) BFs and a sample of S\&P 500 index funds. To obtain our sample of traditional BFs, we dropped all of the funds that we identify as being TDFs, and then restrict the sample to funds where the Lipper objective (as reported in CRSP) is "Balanced Fund." To obtain our sample of S\&P 500 index funds, we first require that the fund name include "S\&P" or "500." Then, we manually drop funds that are not traditional S\&P 500 index funds (e.g., the Direxion Funds S\&P 500 Bear 2.5x Fund).

\section{Characterizing cross-sectional heterogeneity in TDFs}

We start by characterizing the cross-sectional heterogeneity in TDFs. Namely, for each year and target date, we compute statistics summarizing the ex-post heterogeneity in realized returns and alphas. We then turn to statistics meant to capture ex-ante differences in TDF return distributions: the time-series volatility of idiosyncratic returns, and the $R^{2} \mathrm{~s}$ and U.S. equity betas from factor models. For comparison, we also report descriptive statistics for the sample of BFs offered by families that offer TDFs. Given the high market concentration documented in Table 1, we compute both equal-weighted and value-weighted cross-sectional standard deviations of the different 
measures.

Table 2 documents the substantial cross-sectional dispersion in realized annual returns of TDFs during our sample period. ${ }^{17}$ For example, for the $2015-2020$ TDFs, the equal-weighted cross-sectional standard deviation increases from $0.5 \%$ in 2000 to $1.8 \%$ in 2012 . The increase was especially marked between 2007 and 2008, jumping from 2.0\% to 5.1\%. Similarly, the valueweighted standard deviation increases from $0.4 \%$ in 2000 to $1.8 \%$ in 2012 , and jumps from $1.2 \%$ to $3.5 \%$ between 2007 and 2008. The difference between the maximum and the minimum annual returns (range) increases from $1.1 \%$ to $8.5 \%$ between 2000 and 2012 , and from $7.3 \%$ to $27.2 \%$ between 2007 and 2008 .

The patterns are similar for the other four pairs of target dates. In every case, we find that the standard deviation of annual returns is higher in the years after the PPA (2007-2012) than in the years before (2000-2006). Across all five target dates, the equal-weighted standard deviation increases from $1.7 \%$ (pre-PPA) to $2.6 \%$ (post-PPA), and the difference is statistically significant at the one-percent level. The facts that TDFs with the same target date exhibit significant crosssectional dispersion in returns, and that this dispersion increases following the PPA, are the main stylized facts of our study. ${ }^{18}$ The fact that we find the greatest post-PPA return dispersion among TDFs with the earliest target dates suggests that those investors closest to retirement face the greatest uncertainty about TDF returns.

When we switch our focus to BFs, we find that their annual returns exhibit more crosssectional dispersion than the annual returns of TDFs, on average, although in some specific instances the magnitudes are comparable. This is consistent with there being a wider range of investment

\footnotetext{
${ }^{17}$ In order to increase the size of the cross-section for each year, we combine TDFs with adjacent target dates (e.g., 2015 and 2020).

${ }^{18}$ The fact that the changes in dispersion are qualitatively similar using the equal-weighted and value-weighted measures indicates that the heterogeneity that we document is not being driven by a small number of funds with few assets under management. At the same time, the fact that the value-weighted measures are consistently lower than the equal-weighted measures is consistent with our hypothesis that entrants face a greater incentive to generate idiosyncratic returns than existing market leaders.
} 
strategies within the full sample of BFs than within TDFs with any pair of target dates. The more interesting finding is that we cannot reject the null hypothesis that the dispersion in BF returns is the same Pre-PPA as Post-PPA (p-value of 0.566).

In Table 3, we focus on the idiosyncratic component of realized annual TDF returns. To control for the effect of systematic risk on TDF returns, we estimate alpha using a five-factor model and daily excess returns. ${ }^{19}$ We find that there is significant cross-sectional dispersion in the alphas, and that the dispersion is higher in the years after the PPA. Across all five target dates, the equal-weighted standard deviation increases from 1.4\% (Pre-PPA) to $2.0 \%$ (Post-PPA), and the difference is statistically significant at the one-percent level. Because these differences are of the same order of magnitude as in Table 2, it appears that a significant fraction of the dispersion in total returns is being driven by dispersion in idiosyncratic returns. In contrast, although the equal-weighted standard deviation of $\mathrm{BF}$ alphas increases by $0.9 \%$, this difference is not statistically significant at conventional levels (p-value of 0.247), and, unlike with TDFs alphas, we observe no increase in the value-weighted standard deviation of BF alphas.

The analysis above documents significant heterogeneity in realized, or ex-post, TDF returns. Differences in realized returns and alphas must reflect underlying ex-ante differences in asset allocation, security selection, or both. Nevertheless, it is possible that, despite these ex-post differences, the ex-ante distributions of returns for different TDFs were not that different. To address this issue, we switch our focus to the time-series volatilities of idiosyncratic returns. Because the volatilities of idiosyncratic returns describe the statistical properties of TDF returns, they are more likely to reflect ex-ante differences in TDF behavior.

Table 4 reports statistics for idiosyncratic volatilities, estimated as the annualized - scaled

\footnotetext{
${ }^{19}$ The five factors are the daily excess returns of the value-weighted CRSP U.S. market, MSCI World Index excluding the U.S., Barclays U.S. Aggregate Bond Index, Barclays Global Aggregate excluding the U.S., and GSCI Commodity Index. To calculate fund $i$ 's five-factor alpha in month $t$, we estimate the index model in month $t-1$ using daily returns from months $t-12$ to $t-1$. To calculate fund $i$ 's five-factor alpha in year $t$, we compound the alphas obtained from the rolling twelve-month regressions.
} 
by $\sqrt{12}$ - within-TDF standard deviation of monthly five-factor alphas during each calendar year. We then compute yearly summary statistics of the idiosyncratic volatilities across TDFs with the same target date. The patterns are qualitatively similar to those documented in Table 3. Across all five target dates, the idiosyncratic volatility approximately doubles, from $1.1 \%$ to $2.1 \%$. This difference is statistically significant at the one-percent level, while the slightly smaller increase for BFs, from $1.5 \%$ to $2.1 \%$, is not ( $\mathrm{p}$-value of 0.200 ). Note that the serial correlation in idiosyncratic volatilities is 0.515 , which is both economically and statistically significant (p-value of 0.000 ). This increases our confidence that idiosyncratic volatilities capture ex-ante differences in risk-taking.

Table 5 reports statistics for another estimate of ex-ante risk-taking: the $R^{2} \mathrm{~s}$ of the fivefactor model. We estimate the serial correlation in the $R^{2} \mathrm{~s}$ of TDFs to be 0.751 (p-value of 0.000 ), which is even stronger than the serial correlation in idiosyncratic volatilities. Nevertheless, across all five pairs of target dates, we document a decrease in average $R^{2} \mathrm{~s}$ and an increase in the dispersion of $R^{2}$ s. For example, for the 2005-2010 funds, the average $R^{2}$ decreases from $96.3 \%$ in 2001 to $94.7 \%$ in 2012 , whereas the equal-weighted (value-weighted) standard deviation increases from $1.2 \%$ $(0.8 \%)$ to $6.2 \%(4.1 \%)$. Amihud and Goyenko (2013) interpret lower $R^{2}$ s as evidence of greater manager selectivity and show that they are negatively correlated with fund size in their sample of equity funds. In our setting, it appears that the entry of new participants into the TDF market has led to more idiosyncratic volatility as a fraction of the total volatility of TDF returns. Interestingly, this increase in volatility seems to be mainly driven by some funds producing returns with especially low $R^{2}$ s. For the $2005-2010$ TDFs, the lowest $R^{2}$ was $95.3 \%$ in 2001 , but only $64.8 \%$ in 2012 . More generally, the drop in the minimum $R^{2} \mathrm{~s}$ is especially pronounced during the last three years of our sample.

Finally, to capture dispersion in glide paths, we focus on dispersion in the U.S. equity betas. The U.S. equity beta is estimated year-by-year, using daily excess returns, in the same five-factor 
model that we use to estimate TDF alphas. Table 6 presents the summary statistics for beta. Across all five target dates, we find that average U.S. equity betas are significantly lower in 2012 than in 2001. For example, for 2015-2020 TDFs, they fall from 0.58 to 0.46 . This decline is precisely what we expect to observe in a fixed set of glide paths. We also find evidence of increased dispersion in betas in the years after the PPA, however, with the equal-weighted standard deviation increasing from 0.08 to 0.11 (p-value of 0.000 ).

Overall, Tables 2 through 6 reveal that the sample of TDFs in existence after the PPA of 2006 exhibited greater dispersion in ex-post and ex-ante risk than the sample of TDFs in existence pre-PPA. The fact that idiosyncratic volatilities and $R^{2}$ s are highly persistent suggests that it is easier for entrants to offer TDFs that pursue volatile investment strategies than it is incumbents to change the investment strategies of existing TDFs. Therefore, we expect that the change in TDF characteristics is mainly attributable to post-PPA entrants. We test a version of this hypothesis in the next section. ${ }^{20}$

As the last exercise to characterize and benchmark the heterogeneity in TDFs, we decompose the total dispersion in the various TDF measures into what is driven by time variation of the average measure for a TDF with a given target date, and what is driven by cross-sectional variation around the average. The same exercise is then performed for the universes of BFs and S\&P 500 index funds. (See Section A.3 of the Appendix for details.) Regardless of the measure, we find that fund dispersion is highest for BFs and lowest for index funds, with TDFs of all target dates falling in between. Hence, perhaps not surprisingly, TDFs are characterized by more heterogeneity than commodity-like index funds, but less heterogeneity than BFs, which are less constrained in their market-timing and security-selection decisions.

\footnotetext{
${ }^{20}$ Because our regressions include target-date-by-time-period fixed effects, they allow us compare the return dispersion of TDF offered by different families while holding constant the target date and month. These specifications do not, however, allow us to test for time trends in the behavior of incumbent TDFs.
} 


\section{Does TDF heterogeneity reflect optimal risk-taking?}

\subsection{The role of risk-taking incentives}

Our general hypothesis is that heterogeneity in the behavior of TDFs reflects heterogeneity in the incentives faced by the families offering them. To explain the increased dispersion in idiosyncratic returns documented in the previous section, we consider three incentives related to risk-taking. First, because flows into TDFs are likely to be driven by plan sponsor decisions about which TDFs to include on their investment menus (Pool et al. (2013) and Sialm et al. (2015)), we hypothesize that flows into TDFs are more likely to chase risk-adjusted returns than are flows into the typical retail mutual fund. To test this hypothesis, we test whether flows into TDFs are more sensitive to net returns or five-factor alphas, and we compare the flow-performance relations of TDFs and BFs. Evidence that TDFs compete on risk-adjusted returns would help to rationalize dispersion in risk-adjusted returns.

Second, by increasing demand for TDFs as default investment options, the PPA significantly increased the future share of retirement plan assets that will be invested in TDFs. As a result, the PPA increased the incentive of mutual fund families to place their TDFs on retirement plan investment menus. Because we cannot observe the counterfactual market structure, we cannot quantify the strength of this incentive. TDFs were, after all, gaining market share before the PPA. Nevertheless, the passage of the PPA likely helps to explain why, in Table 1, we observe 17 families entering the TDF market in 2007 and 2008, increasing the total from 27 to 44 . The large number of entrants is likely to intensify competition for market share.

Third, there is a well-established literature arguing that mutual funds facing more convex payoffs are more likely to engage in risk-taking (e.g., Brown, Harlow, and Starks (1996) and Evans (2010)). In our setting, variation in convexity arises from the fact that entrants have the fewest 
assets - and therefore fewer management fees - to lose if they underperform their peers. This fact leads us to hypothesize that families entering the market for TDFs after the PPA will pursue more idiosyncratic strategies than existing families. ${ }^{21}$ Combining the three risk-taking incentives leads us to predict that the increased dispersion in idiosyncratic returns documented in Tables 2 through 5 is driven by the large number of families with low TDF market shares. Moreover, we predict that the risk-taking incentives are especially large for families that entered the market after 2006. This is because low-market-share incumbents typically have more assets at stake than entrants, and because incumbents have less flexibility to pursue idiosyncratic investment strategies given the glide paths and fund holdings they previously disclosed in their prospectuses.

Before testing these predictions, it is helpful to consider several possible refinements. One refinement concerns the potential mechanism. One possibility is that, following the PPA, new entrants are more likely to assign funds pursuing more idiosyncratic strategies to their TDFs. In other words, they might be more likely to design TDFs that behave like BFs. Alternatively, it could be that, following the PPA, families with more idiosyncratic investment strategies are more likely to enter the TDF market. While this is not a crucial distinction from the investor's perspective, we can shed light on the mechanism by comparing specifications that do and do not control for the behavior of a family's BFs. We can also ask whether the correlation between the return characteristics of a family's TDFs and BFs is stronger for entrants than for incumbents. This is what we would expect if incumbents reduce TDF risk levels below BF risk levels, but entrants do not.

A separate issue is that families face a choice about when to enter the market and pursue an idiosyncratic investment strategy. To the extent that pursuing the volatility option this year prevents families from pursuing the volatility option next year, the incentives of entrants to both

\footnotetext{
${ }^{21}$ An earlier version of our study developed a simple model formalizing this intuition. We assumed that fund families care about both the expected level and the volatility of flows and that, because a TDF can at most lose the assets currently under management, expected flows can be increasing in the volatility of returns. As a result, new TDFs may find it optimal to load on risk as the effect on expected flows more than offsets the effect on the volatility of flows.
} 
enter the market immediately following the PPA and pursue idiosyncratic strategies may be weaker than we claim. As a practical matter, fund families appear to have decided not to delay the entry and the possible exercise of the volatility option: we observe 17 entrants between 2007 and 2008, and only 3 entrants between 2009 and 2012. Our conjecture is that families expected that it would be easier to have their TDFs added to plan menus in the years immediately following the PPA, than to convince plan sponsors to replace previously-chosen TDFs with new TDFs. In other words, "stickiness" in the choice of TDF by plan sponsors may have fully offset any possible benefit from waiting to exercise the volatility option.

There is another well-established literature showing that the flow-performance relation for retail mutual funds is convex (e.g., Sirri and Tufano (1998)). This literature prompts us to test for convexity in the flow-performance relation of TDFs that is unrelated to market share. Finally, there is an an interesting question about how the costs and benefits of pursuing an idiosyncratic return strategy vary with market shares in other markets. On the one hand, a family with low market share in the overall mutual fund market might have a difficult time attracting the attention of plan sponsors, limiting the potential benefit of pursuing idiosyncratic returns. On the other hand, a family with high market share in the overall mutual fund market might worry that abnormally low TDF returns will damage its reputation with plan sponsors and retail investors. These possibilities prompt us to estimate additional specifications that test whether risk-taking incentives related to TDF market share vary with overall market share. 


\section{$5.2 \quad$ Flows and performance}

In this section, we test the hypothesis that TDF flows respond to risk-adjusted returns. In Table 7, we estimate the following flow-performance model:

$$
\text { flow }_{i j t}=a_{j}+b_{t}+c^{\top} X_{j t}+d^{\top} Z_{i j t}+\epsilon_{i j t},
$$

where flow $_{i j t}$ is the one-year net flow, measured as a percentage of assets under management at the beginning of the period. The specification is motivated by the flow-performance regression in Del Guercio and Reuter (2014), who also run a horse race between raw and risk-adjusted returns, but is extended to capture features of the TDF market. The $X_{j t}$ vector includes the natural logarithm of the total number of funds with target date $j$ in year $t$, which is a measure of the degree of competition for flows. In the full specification, the $Z_{i j t}$ vector includes: the one-year realized (net) return in year $t-1$; the one-year (net) alpha in year $t-1$; the volatility of monthly fund returns in year $t-1$; the net flow into fund $i$ in year $t-1$; a dummy equal to one if the fund was introduced after December 2006; a dummy equal to one if the fund was introduced by a family that entered the TDF market after December 2006; the fund-level expense ratio measured in year $t$; the natural logarithm of fund assets under management in year $t-1$; and the natural logarithm of family assets under management in year $t-1$. Specifications with TDF flows as the dependent variable include both calendar-year fixed effects and target-date fixed effects. Specifications with balancedfund flows only include calendar-year fixed effects. Standard errors are simultaneously clustered on mutual fund family and year.

Our main finding is that flows into TDFs chase lagged risk-adjusted returns rather than raw

returns. In the first column, which includes the smallest set of controls, we find that a $1 \%$ increase in alpha in year $t-1$ is associated with an increase in percentage flows of $2.430 \%$. In the third column, 
which includes the full set of controls (including lagged flows), the estimated coefficient on lagged alpha is $1.807 \%$. In each column that includes both net returns and alphas, the estimated coefficient on raw returns is much smaller in value (between $0.159 \%$ and $0.361 \%$ ), statistically indistinguishable from zero, but statistically significantly different from the estimated coefficient on alpha ( $\mathrm{p}$-values between 0.001 and 0.015 ). These estimates imply that plan sponsors (or their consultants) reward risk-adjusted returns rather than raw returns, a finding which helps to rationalize the observed dispersion in idiosyncratic returns. ${ }^{22}$ We also find that flows are decreasing in the lagged volatility of monthly returns. ${ }^{23}$ Both sets of findings are broadly consistent with Del Guercio and Tkac (2002), who conclude that pension funds face fundamentally different flow-performance relations than retail mutual funds.

When we re-estimate the full specification on the sample of BFs, we find that flows respond to both raw and risk-adjusted returns. ${ }^{24}$ This is the same general pattern that Del Guercio and Reuter (2014) find in a pooled sample of domestic equity funds. The estimated coefficient on the lagged alpha is approximately half of that estimated for TDFs, and statistically indistinguishable from the estimated coefficient on the lagged raw return. In addition, while lagged flows continue to predict current flows, the volatility of monthly returns does not. These differences strengthen our conclusion that the flow-performance relation for TDFs differs from that of the typical retail mutual fund, resulting in a different set of risk-taking incentives.

Finally, we use a series of dummy variables to measure the convexity of the flow-performance

\footnotetext{
${ }^{22}$ When we estimate a version of this specification based on three-year flows and contemporaneous three-year returns and alphas, the estimated coefficients on both return measures are positive and statistically significant, but the estimated coefficient on alpha is approximately three times larger (14.853\% versus $5.454 \%$ ) and the two coefficients are statistically significantly different at the 5 -percent level.

${ }^{23}$ When we estimate specifications that control instead for the lagged volatility of monthly alphas, the coefficients are small and positive, with very large standard errors.

${ }^{24}$ In contrast, Sandhya (2011) documents that quarterly TDF flows - likely dominated by the behavior of DC investors - are insensitive to past quarterly performance, whereas balanced-fund flows-likely dominated by the behavior of non-DC investors - are sensitive to past quarterly performance. The fact that we find different patterns when we focus on longer-horizon returns suggests that the appropriate horizon to evaluate the flow-performance relation may be different for funds catering to DC and non-DC investors.
} 
relation for TDFs. The fourth-quartile dummy variable indicates whether fund $i$ 's five-factor alpha is in the top quartile of five-factor alphas earned in year $t-1$ by TDFs with the same target date; the other dummy variables are defined similarly. The flow-performance relation that we estimate for TDFs is less convex than has been estimated for many types of retail funds, but is qualitatively similar to that estimated in Sialm et al. (2015). We find that top-quartile funds grow by an additional $7.0 \%$ and that bottom-quartile funds shrink by $7.9 \%$; there are no additional inflows or outflows for the middle quartiles. This symmetry implies that expected flows should not be much affected by the volatility of risk-adjusted returns. In turn, this implies that risk-taking incentives are only likely to arise when families have few assets to lose.

\subsection{Explaining the cross-sectional dispersion in TDF returns and alphas and the level of idiosyncratic risk}

Having found that TDFs flows respond to risk-adjusted returns, we next test the hypothesis that increased competition following the PPA helps to explain the increased heterogeneity in TDF returns (see Table 8 ). We begin with the regression model:

$$
\left(r_{i j t}-\bar{r}_{j t}\right)^{2}=a_{j t}+b^{\top} X_{i j t}+\epsilon_{i j t},
$$

where $r_{i j t}$ is the monthly return of TDF $i$ and $\bar{r}_{j t}$ is the cross-sectional average return of TDFs with target date $j$ in month $t$; $a_{j t}$ is a target-date-specific fixed effect for month $t$; and $X_{i j t}$ is a vector of covariates intended to capture family-level incentives and investment strategies. This vector includes: dummy variables equal to one if the market share of family $j$ 's TDFs was $\leq 1 \%$ ("Low Market Share") or $>1 \%$ and $\leq 5 \%$ ("Medium Market Share") in month $t-1$; dummy variables equal to one if family $k$ entered the TDF market before or after December 31, 2006 ("Pre-PPA Family" versus "Post-PPA Family"); interactions of the Low Market Share dummy variable with 
the Pre-PPA and Post-PPA dummy variables; a dummy variable equal to one if TDF $i$ invests in index funds; and the average comparable measure of return dispersion for BFs in TDF $i$ 's family. ${ }^{25}$ We expect TDFs investing in index funds to exhibit less cross-sectional return dispersion than TDFs based on actively managed funds. To the extent that some families pursue more volatile investment strategies across their full range of funds, we also expect the average cross-sectional return dispersion of a family's BFs to be positively correlated with the cross-sectional return dispersion of its TDFs. By including a separate fixed effect for each target-date-month pair, we are comparing the return dispersion of different TDFs with the same target date in the same month. Consequently, whereas the patterns in Tables 2-6 partially reflect the effect of the financial crisis on all TDF returns, the coefficients in equation (2) are being identified purely by cross-sectional variation in the variables of interest.

In 2006, there are 16 families with Low Market Share, five families with Medium Market Share, and four families with High Market Share (the omitted category in our regressions). In 2012, those numbers are 25, nine, and three, respectively, with three Pre-PPA families and one Post-PPA family rising from Low Market Share to Medium Market Share range, and one PrePPA family falling from High Market Share to Medium Market Share. ${ }^{26}$ Our two main (related) predictions are that families with Low Market Share will pursue more volatile investment strategies than families with Medium or High market share, and that this incentive will be particularly strong for families drawn into the market by the PPA. Table 8 provides support for both predictions. In the baseline specification (column one), we find that dispersion of monthly net returns increases by $0.625 \%$ when TDFs are offered by Low Market Share families. ${ }^{27}$ In column two, where we add the

\footnotetext{
${ }^{25}$ The specification is significantly different from that estimated in Table 7 because our focus has shifted from investor and plan-level decisions about how to allocate retirement assets to family-level decisions about risk-taking as a function of TDF market share.

${ }^{26}$ In Table A.2, we report the number of families that fall into six different categories based on their TDF market share (low, medium, and high) year of entry (pre-PPA and post-PPA). We also report the number of TDF-month observations for each type of family in each calendar year.

${ }^{27}$ We estimate this effect by taking the square root of the regression coefficient 0.391 .
} 
Post-PPA family dummy variable, we see that about $60 \%$ of the Low Market Share effect is actually a Post-PPA family effect. And, in column four, where we allow the Low Market Share effect to differ for Pre-PPA and Post-PPA families, we find that almost all of the Low Market Share effect is driven by the TDFs of families entering the market post-PPA. The dispersion of monthly returns is $0.884 \%$ higher for Post-PPA families (p-value of 0.026 ), versus $0.336 \%$ higher for Pre-PPA families (p-value of 0.129), and the difference is statistically significant at the ten-percent level. Our findings are quantitatively similar when we run family-level regressions that focus on the average level of return dispersion in family $k$ in month $t$, which is reassuring because the typical family's TDFs allocate different amounts to the same underlying sample of funds.

In the remaining columns, we estimate analogous fund-level and family-level specifications for two other dependent variables: cross-sectional dispersion in the five-factor alpha in month $t$, and the (non-annualized) idiosyncratic volatility of TDF $i$ in year $t$. We again find consistent evidence that among Low Market Share, Post-PPA families generate more volatile alphas than Pre-PPA families. In addition, we now find that both types of Low Market Share families generate more volatile alphas than High Market Share families. For example, in the fund-level regression for the cross-sectional dispersion in five-factor alphas, the estimated effects are $0.272 \%$ for PrePPA families and $0.528 \%$ for Post-PPA families, and both effects are statistically significant at the 5-percent level.

Although the coefficient on the index-fund-based TDF dummy variable is consistently negative, it is only statistically significant when the dependent variable is idiosyncratic volatility. A more robust relation is between the dispersion of TDF returns and the average dispersion of returns on BFs within the same family, suggesting that some families pursue systematically more volatile investment strategies than others. Adding this family-level control significantly increases $R^{2}$ (from $10.94 \%$ in column two to $16.08 \%$ in column three), but only has a modest effect on the Low Market 
Share and Post-PPA coefficients. In other words, while the PPA appears to have drawn families with more idiosyncratic investment strategies into the TDF market, we find significantly higher dispersion in risk-taking on the part of Post-PPA TDFs with low market share, even after controlling for this family-level trait. ${ }^{28}$

Although our tests focus on a family's share of the TDF market, we acknowledged in Section 5.1 that the expected costs and benefits of increasing idiosyncratic risk may also depend on the family's share of the overall mutual fund market or 401(k) market. Specifically, families with the lowest overall market shares may have the least to gain from pursuing an idiosyncratic return strategy, because consultants may still be reluctant to add them to retirement plan menus. Families with the highest overall market shares, on the other hand, may have the most to lose if abnormally low TDF returns damage their reputation with plan sponsors. We test these predictions in an alternative version of Table 8, which we include in the Supplementary Appendix. One set of specifications includes dummy variables indicating low or medium market share in the overall mutual fund market (based on total assets under management in CRSP), rather than in the TDF market. Another set of specifications interact the dummy variables indicating low, medium, and high market share in the TDF market with dummy variables indicating low, medium, and high market shares in the overall mutual fund market. We find the strongest evidence of risk-taking by families that have a low TDF market share and a medium overall market share. But, we also continue to find significantly more risk-taking by Post-PPA families, the majority of which have low TDF market share. We conclude that the risk-taking incentives associated with entering the TDF market after the PPA are even stronger for those families with a medium market share in the

\footnotetext{
${ }^{28}$ In untabulated results, we document that the cross-sectional dispersion of returns and alphas is comparable for pre- and post-PPA families. However, while for pre-PPA families the cross-sectional dispersion of TDF returns and alphas is significantly lower than that of BF returns and alphas, this is not true for post-PPA families, for which TDF and $\mathrm{BF}$ returns and alphas have comparable cross-sectional dispersion. Thus, it appears that one channel through which post-PPA families responded to their risk-taking incentive was by choosing not to adjust the idiosyncratic risk of their TDFs down relative to their BFs.
} 
overall mutual fund market.

\subsection{Explaining differences in the level of factor-model $R^{2} \mathrm{~s}$}

As an alternative measure of risk, we turn to factor-model $R^{2}$ s (see Table 10). We consider two factor models: a single-factor model, with the U.S. equity excess return as the only factor ("CAPM"), and the five-factor model used through the paper ("five-factor model"). The fund-level and familylevel regressions mirror those discussed in the previous section, except that, because the unit of observation switches from month to year, we focus on market shares calculated in month $t-12$. In the fund-level regressions, the dependent variable is the $R^{2}$ of TDF $i$ in year $t$, minus the equalweighted average $R^{2}$ for all TDFs with the same target date. ${ }^{29}$ In the family-level regressions, it is the equal-weighted average of these deviations for family $k$ in year $t$.

Since lower $R^{2}$ s are associated with more idiosyncratic returns, our prediction is that PostPPA families with Low Market Share will have the lowest $R^{2}$ s. In Table 9, we find evidence consistent with this prediction. The $R^{2} \mathrm{~s}$ of Low Market Share, Post-PPA families are between $5.5 \%$ and $6.2 \%$ lower when we focus on the one-factor model, and between $2.7 \%$ and $3.0 \%$ lower when we focus on the five-factor model. All of these differences are statistically significant at the five-percent level from the omitted category of High Market Share families. In terms of economic significance, the differences in five-factor $R^{2}$ are larger than almost all of the equal-weighted crosssectional standard deviations that we report in Table 5 for 2007-2009, and approximately half the size of the average equal-weighted cross-sectional standard deviation in 2010-2012. We continue to find that the return properties of a family's BFs help to predict the return properties of its TDFs.

\footnotetext{
${ }^{29}$ Since we include target-date-by-year fixed effects, subtracting the cross-sectional average does not affect our estimates in the fund-level regressions. De-meaning is necessary, though, when calculating the dependent variable used in the family-level regressions.
} 


\subsection{Explaining differences in the levels and the dispersion in five-factor-model betas}

In the previous two sections, we found that competition for TDF flows focuses on risk-adjusted returns, and that families entering the market after the PPA responded by generating more idiosyncratic returns. In this section, we test for differences in betas. The fund-level and family-level regressions in Table 10 mirror those in Table 9. To the extent that plan sponsors focus primarily on risk-adjusted returns, we do not expect entrants to offer TDFs with systematically higher or lower betas than other families. On the other hand, because entrants may find it difficult to market their TDFs to plan sponsors if they have the same glide paths as the market leaders, we expect entrants to increase dispersion in betas. (In the next section, we test whether dispersion in betas results in better risk matching between TDFs and firms.)

The dependent variable in Panel A is the beta of TDF $i$ in year $t$ minus the equal-weighted average of all TDFs with the same target date, or the equal-weighted average deviations for TDF $i$ 's family in year $t$. In this panel, positive coefficients imply positive tilts in beta. The dependent variables in Panel $\mathrm{B}$ are the squared deviation for TDF $i$ in year $t$ or the equal-weighted average squared deviation for TDF $i$ 's family in year $t$. Here, positive coefficients imply greater dispersion in beta. We again control for whether TDF $i$ is based on index funds (or the fraction of family TDF assets that are based on index funds), as well as the average beta tilt or dispersion within TDF $i$ 's family. ${ }^{30}$

We find some evidence that Low Market Share, Post-PPA families have higher loadings on U.S. debt, global debt, and commodities. However, the main finding in Panel A is that the beta tilts of TDFs are strongly positively correlated with the beta tilts of a family's BFs. The effect is especially large for the exposure to the commodity factor, where a 0.10 increase in the

\footnotetext{
${ }^{30}$ Because we are estimating specifications for five separate betas, we omit the specifications that include Low Market Share and Post PPA Family dummies without an interaction.
} 
commodity betas of BFs is associated with a 0.06 increase in the commodity betas of TDFs. While it is perhaps not surprising that families make similar asset allocation decisions in their TDFs and traditional BFs, the typical investor is unlikely to know whether her TDF is from a family that has above-average allocations to global equity or commodities.

The main finding in Panel B is that Low Market Share, Post-PPA families offer TDFs with more disperse betas with respect to U.S. equity, global equity, global debt, and commodities. This suggests that the movement into riskier asset classes documented in Elton et al. (2014) is being driven by families entering the TDF market following the PPA.

\section{Does TDF heterogeneity reflect risk matching?}

\subsection{The role of risk-matching incentives}

To explain the increased dispersion in betas, we consider incentives related to risk matching. The Department of Labor now explicit encourages plan sponsors to take worker characteristics into account when choosing TDFs. ${ }^{31}$ Dispersion in glide paths should be readily observable to plan sponsors and their consultants. Therefore, one hypothesis is that "risky" firms will pick safer TDFs for their 401(k) plans than "safe" firms. For example, firms in riskier industries may avoid TDFs with larger-than-average allocations to equity, and this may be especially true when the plans feature automatic enrollment, since the TDFs in these plans are likely to be the default investment options. This form of risk matching implies a negative correlation between TDF risk and firm risk. ${ }^{32}$

\footnotetext{
${ }^{31}$ In a 2013 memo directed at plan fiduciaries, the DOL writes: "You should consider how well the TDFs' characteristics align with eligible employees ages and likely retirement dates. It also may be helpful for plan fiduciaries to discuss with their prospective TDF providers the possible significance of other characteristics of the participant population, such as participation in a traditional defined benefit pension plan offered by the employer, salary levels, turnover rates, contribution rates and withdrawal patterns." U.S. Department of Labor, Employee Benefits Security Administration, "Target Date Retirement Funds - Tips for ERISA Plan Fiduciaries," February 2013.

${ }^{32}$ Viceira (2009) notes that: "Employees with volatile labor earnings or labor earnings that are highly correlated with equity returns should avoid investing in the current generation of life-cycle funds, which exhibit significant equity tilts. For these investors, their human wealth is less 'bond-like' and more 'equity-like.' Therefore they already have exposure to equities through their human wealth and should avoid excessive exposure, or any exposure at all, to equities in their portfolios. Since the correlation of labor earnings with stock returns is likely to be similar for
} 
The alternative hypothesis is that, if the risk aversion of the representative employee varies across firms (Berk et al. (2010)), and if different firms appeal to employees with different levels of risk aversion, then TDF risk and firm risk are positively correlated. ${ }^{33}$ We test for non-zero correlations using plan-level data and measures of systematic and idiosyncratic risk.

\subsection{Testing for risk matching in plan-level data}

To test the risk-matching hypothesis, we analyze retirement plan-level data from BrightScope. ${ }^{34}$ The full database covers 16,766 distinct 401(k) and 403(b) plans, offered by 15,403 distinct firms, in 2010. There are more plans than firms because some firms offer multiple plans. For example, United Airlines offers separate retirement plans for its pilots and ground employees. Firm-level data include the firm's name, primary address, and 6-digit North American Industry Classification System (NAICS) code. We are able to locate a ticker and estimate a CAPM beta for 1,740 of the firms in the BrightScope database. ${ }^{35}$ Plan-level data include assets under management, number of participants, whether the plan offers company stock, whether the plan has auto enrollment, whether the plan has a single record keeper (SRK), and the identity of the record keeper. Investment-level data include the name and type (mutual fund, collective trust, separate account, company stock, etc.) of each investment option offered by each plan, whether the investment option is a TDF, and the total dollars invested in the option.

employees within the same industry or company, these considerations suggest that there is a benefit to the creation of industry-specific or company-specific life-cycle funds." Bagliano et al. (2013) compute the utility costs of ignoring heterogeneity in labor income variance in constructing TDFs; see Section 7.

${ }^{33}$ Viceira (2009) suggests that: "Mutual fund companies might want to consider offering life-cycle funds that exhibit different equity tilts. That is, they might want to offer 'conservative,' 'moderate,' and 'aggressive' life-cycle funds. These funds will help capture investor heterogeneity in risk tolerance." Gomes et al. (2008) compute the utility costs of ignoring heterogeneity in risk aversion in constructing TDFs; see Section 7.

${ }^{34}$ Because BrightScope must hand collect data on investment menus, our sample is skewed toward firms with larger 401(k) or 403(b) retirement plans. A comparison of our sample to Form 5500 filings of plans with at least $\$ 1$ million in assets suggests that BrightScope covers $78.4 \%$ of all defined contribution retirement plan participants in 2010 and $89.3 \%$ of all defined contribution retirement plan assets.

${ }^{35}$ We use the 24 monthly returns between December 2007 and November 2009 to estimate the CAPM beta as of December 2009. Our proxy for the market portfolio is the excess return on the market as reported on Ken French's website. For comparability, we use the sample time period and market portfolio to estimate the CAPM beta of each mutual fund in the BrightScope sample. 
Summary statistics for the BrightScope data set are presented in Table 11. Approximately $66 \%$ of the plans offer some form of TDF, with $50 \%$ offering TDF mutual funds. When we count TDFs with different target retirement dates as a single investment option, TDFs represent $2.7 \%$ of the investment options and $9.7 \%$ ( $\$ 242$ billion) of the $\$ 2,495$ billion in assets under management in our sample of plans in $2010 .{ }^{36}$ The fact that TDFs managed almost $10 \%$ of defined contribution retirement plan assets in 2010 highlights the important role that TDFs have come to play in retirement wealth accumulation.

The advantage of using plan data from 2010 to test for risk matching is that plan sponsors were able to choose from the full range of TDFs introduced following the PPA. Table 11 reveals considerable dispersion in firm risk, whether measured by the CAPM beta or the standard deviation of residual returns. Consistent with our earlier analysis, it also reveals significant dispersion in the CAPM betas of the TDFs offered within the plans. For example, the estimated CAPM betas of 2020 TDFs range from 0.63 to $1.00 .^{37}$

Within our sample, there are 7,687 retirement plans that offer TDFs and employ an SRK that is also an asset management firm. When we distinguish investment options managed by SRKs from investment options managed by other asset management firms, we find that $76 \%$ of TDFs are managed by SRKs versus 39\% of non-TDFs. The fact that plan sponsors disproportionately offer the TDFs of their record keepers is prima facie evidence against significant risk matching. ${ }^{38}$

To formally test for a correlation between the riskiness of a firm and the riskiness of the

\footnotetext{
${ }^{36}$ When we focus only on mutual funds, TDFs account for $3.0 \%$ of the investment options and $13.9 \%$ ( $\$ 157$ billion) of the $\$ 1,131$ billion in assets under management.

${ }^{37} \mathrm{It}$ is worth noting that this range of beta estimates contrasts with what reported in Table 6, where 2015-2020 TDF betas range from 0.14 to 0.66 . The reason for the discrepancy is that betas here are estimated from a single-factor, rather than a five-factor model.

${ }^{38}$ There is little evidence that the choice of record keeper reflects concerns about risk matching. Regressions of firm-level risk on record keeper fixed effects result in adjusted $R^{2}$ s between only $0.91 \%$ and $1.51 \%$.
} 
TDF that the firm offers to its employees, we estimate the following cross-sectional model:

$$
\mathrm{TDF} \operatorname{risk}_{i j k}=a+b \text { firm } \operatorname{risk}_{j}+c^{\top} X_{i}+\epsilon_{i j k}
$$

where TDF risk ijk $_{k}$ measures of the risk of the TDF offered in plan $i$ sponsored by firm $j$ in industry $k$, and firm risk $_{j}$ measures the risk of the plan sponsor. If there is any form of risk matching, the estimated coefficient on firm risk ${ }_{j}$ will be non-zero. The $X_{i}$ vector includes several plan-level controls. Because plan sponsors may focus more on TDF risk when plans feature auto enrollment, we include a dummy indicating if the plan features auto enrollment and, in some specifications, an interaction between the measure of firm risk and the dummy indicating if the plan has auto enrollment. Because we find that plans are more likely to offer the TDFs of their record keepers, we include either a dummy equal to one if plan $i$ has an SRK, or the market share of the SRK's investment options in the BrightScope sample. ${ }^{39}$ Our measure of plan-level risk is the average risk of the non-TDF mutual fund options. We also include the natural logarithm of plan assets, the natural logarithm of plan participants, a dummy indicating if the plan offers company stock. In some specifications, we include a separate fixed effect for each industry (defined using the first 3 digits of the NAICS code), to control for average differences in firm risk across industries. Because we were only able to estimate risk for TDFs that are mutual funds, we restrict the sample to plans that offer TDFs that BrightScope classifies as mutual funds (and for which we could obtain mutual fund return data from CRSP). ${ }^{40}$ Standard errors are clustered on industry.

We report the regression results in Table 12. Because we find above that heterogeneity in realized returns is larger for TDFs whose target retirement year is closer, our main measure of

\footnotetext{
${ }^{39}$ The correlation between the market share of an SRK's investment options and the market share of its TDF options is 0.982 , further justifying our earlier focus on a family's market share in the TDF market.

${ }^{40}$ Our findings are unchanged if we expand the sample to include separate accounts and collective trusts and assume that all of the TDFs offered by a given family have the same level of risk.
} 
TDF risk is the CAPM beta of the TDF with a target retirement date of 2020. Our main measure of firm risk is the CAPM beta on firm $j$ 's equity, which limits our sample to 896 plans offered by publicly-traded firms. Within this sample, the estimated regression coefficients on firm risk are negative, but they are neither statistically nor economically distinguishable from zero. Moreover, the modest increase in adjusted $R^{2}$ when we introduce industry fixed effects (from $2.99 \%$ to $6.06 \%$ ), suggests limited matching of TDF risk to average industry risk. Among the other variables, we find that TDF risk decreases with plan assets and increases with the number of plan participants, but neither effect is economically large.

The more interesting findings are that TDF risk is lower in plans that offer a SRK and, within this sample of plans, is decreasing in the market share of the SRK. These findings are consistent both with plan sponsors offering the TDFs of their record keepers and with highermarket-share record keepers offering less risky TDFs than their lower-market-share competitors. A one standard deviation increase in the market share of the SRK is associated with a reduction in CAPM beta of 0.011, while moving from the lowest market share to the highest market share is associated with a reduction of 0.026 . By way of comparison, the standard deviation of TDF betas is 0.058 .

When we instead measure firm-level risk as the median CAPM beta of firms in the same industry, we are able to increase the sample to 7,124 plans. ${ }^{41}$ Within this larger sample, we find a weak positive correlation between the CAPM beta of the TDF and the CAPM beta of the industry. The estimated coefficient of 0.012 implies that a one-standard deviation increase in industry risk is associated with an increase of 0.011 in TDF beta. When we estimate a specification that allows the correlation between TDF risk and firm risk to vary with automatic enrollment, we find that the negative coefficient on the interaction term is similar in magnitude to the positive coefficient

\footnotetext{
${ }^{41}$ When we regress a firm's CAPM beta on a separate fixed effect for each 3-digit NAICS code, the adjusted $R^{2}$ is $20.13 \%$. By way of comparison, when we regress a firm's CAPM beta on a separate fixed effect for the state in which a plan is located, the adjusted $R^{2}$ is $3.93 \%$.
} 
on firm risk. Consequently, we find a weak positive correlation between TDF risk and firm risk in the sample of plans without automatic enrollment and no correlation in the sample of plans with auto-enrollment.

To explore the possibility that riskier firms offer investment menus skewed toward riskier funds, the final specification controls for the average CAPM beta of the plan's non-TDF mutual funds (measured relative to other funds with the same investment objective). The estimated coefficient on this measure of plan risk is positive and statistically significant at the ten-percent level, suggesting that plans offering riskier-than-average non-TDFs also offer riskier-than-average TDFs, but the estimated coefficients on the other variables are unchanged.

In the remaining regressions, we shift our focus from systematic risk to idiosyncratic risk (see Table 12, Panel B). Specifically, we use each firm's and TDF's estimated CAPM beta to decompose its monthly returns into systematic and idiosyncratic components. We then calculate the standard deviation of the idiosyncratic returns over the prior 24 months. (Because the mean of the dependent variable is only 0.010 , for ease of comparison, we multiply the estimated coefficients by 100.)

We find evidence of risk matching in the industry-level regressions. Firms in industries with more idiosyncratic stock returns are more likely to offer TDFs with lower idiosyncratic returns, but the effects are economically modest. A one standard deviation in industry risk is predicted to decrease TDF risk by 0.037 standard deviations. In contrast, a one standard deviation in the market share of the SRK is predicted to decrease TDF risk by 0.22 standard deviations, while moving from the lowest market share to the highest market share is associated with a reduction of 0.54 standard deviations. In other words, our main finding continues to be that record keepers with high market share offer less risky TDFs. We also continue to find that plans offering riskierthan-average non-TDFs also offer riskier-than-average TDFs. 


\section{Heterogeneity in TDF returns: why should we care?}

The empirical analysis of this study has uncovered large differences in ex-post returns - both total and idiosyncratic - for TDFs with same target date. We have also documented substantial differences in estimates of the ex-ante properties of the distribution of returns - idiosyncratic volatilities, $R^{2} \mathrm{~s}$, and betas. Moreover, we have been able to relate these differences to the risk-taking incentives of the fund families offering the funds, but not to the characteristics of the firms whose plans offer the TDF.

At this point, a crucial question is whether the differences in TDFs that we document have the potential to be material to TDF investors. Existing studies suggest that they do. ${ }^{42}$ For example, Gomez et al. (2008) consider the optimal TDF for investors with constant relative risk aversion (CRRA), initially at age 21 and who retire at and 65, and whose life-time expected wage profile and wage volatility is realistically calibrated. They perform the exercise of constraining an investor with an RRA coefficient of eight to follow the average optimal asset allocation path of an investor with an RRA coefficient of five. They estimate this cost at about $234 \%$ of his first-year labor income. Bagliano et al. (2013) consider the appropriateness of a typical TDF for a similar CRRA investor, who may have either normal or high labor income variance. Whereas the typical TDF is nearly optimal for the investor with normal labor income variance, it generates a cost as high as $31 \%$ of the constant consumption level for an investor with high labor income variance and an RRA coefficient of eight. Pang and Warshawsky (2009) do not compute utility costs, but characterize the heterogeneity in outcomes from investing in TDFs with the glide paths followed by the providers in this market. Simulations show that the standard deviation of terminal wealth for an investor who starts investing at age 25 and retires at 65 can differ by as much as $20 \%$, depending

\footnotetext{
${ }^{42}$ In Section A of the Supplementary Appendix we provide our own "back-of-the-envelope" calculations of the costs generated by deviations from optimality. As documented in existing studies, these costs can be substantial and these costs can increase if the properties of returns are not known with certainty.
} 
on the glide path followed by the chosen TDF.

The studies above focus on TDFs that are essentially collections of index funds. Our analysis has shown that a large component of the heterogeneity in TDF returns is due to heterogeneity in idiosyncratic returns. The presence of idiosyncratic returns has the potential to generate additional utility costs: the idiosyncratic risk may not be properly accounted for, and, even if it is accounted for, can generate sizable utility cost unless compensated by extra performance. ${ }^{43}$

Finally, there is the issue of transparency of the risk properties of TDFs. Investors and plan sponsors should know the risk - both systematic and idiosyncratic - that they are exposed to when investing in a TDF. Indeed, the introduction of TDFs has been predicated on two main grounds (see, for example, the quotes in Section A.2 of the Appendix): first, investors who select TDFs are less likely to be exposed to too little systematic equity risk when young, and too much systematic equity risk when old; second, investors who invest in TDFs are less tempted to rebalance their portfolio in response to recent returns. If TDF have unclear risks, it is possible that investors choosing TDFs are no better off than they would be by choosing investments on their own. Moreover, as the reputation of TDFs is tarnished, investors may become more reluctant to invest in TDFs.

The arguments above are strengthened by the consideration that the majority of 401(k) plans limits their participants' choice to the TDFs offered by a single family. Hence, the costs that heterogeneity in TDFs may generate cannot be easily diversified away by investing in many different TDFs.

\section{Conclusions}

The market for TDFs is important for at least two reasons: First, because TDFs are a relatively new financial product, this market allows us to study how mutual fund families structure new

\footnotetext{
${ }^{43}$ See Section A of the Supplementary Appendix for some calculations.
} 
investment products and compete for market share. Second, given the widespread, legislationinduced use of TDFs as default investments in DC retirement plans, this is a market with special policy significance.

We document pronounced heterogeneity in the TDF universe: TDFs with the same target date have delivered very different returns to investors. The heterogeneity of returns has increased over time, with the large number of families entering the market after the passage of the PPA pursuing more idiosyncratic investment strategies. Because we show that flows into TDFs respond to alpha rather than total returns, these patterns are consistent with new entrants responding to their incentives to attract retirement plan sponsors by generating higher idiosyncratic returns. On the other hand, we find little evidence that the heterogeneity in risk-taking that we document is driven by TDFs catering to different risk clienteles. As argued in the existing literature, this lack of matching between the risk properties of the TDFs offered in a given firm's plan, and the risk characteristics - human capital risk and risk preferences - of the firm's employees has the potential to generate substantial utility costs.

Our findings suggest that the widespread adoption of TDFs will not necessarily equalize the returns earned by investors enrolled in different 401(k) plans. Indeed, the cross-sectional dispersion in returns of funds with 2015-2020 target dates was so large in 2008 and 2009, that it came to the attention of regulators. On November 29, 2010, regulation was proposed to increase investor understanding of how TDFs operate. Specifically, TDFs would be required to provide: i) a description and graphical illustration of the asset allocation, how it will change over time, and the point when it will be the most conservative; ii) a clarification of the relevance of the date (if the name includes a target date) and the target age group for which the investment is designed; and iii) a statement that a participant is not immune from risk of loss, even near or after retirement, and 
that no guarantee of sufficient returns to sustain an adequate retirement income can be given. ${ }^{44}$

The pronounced heterogeneity in realized TDF returns that we document means that a well-informed $410(\mathrm{k})$ investor, who is typically limited to the TDFs of a single mutual fund family, may face returns that depart significantly from the industry average. Importantly, these differences in returns are largely driven by differences in alphas and cannot be anticipated based on disclosed differences in glide path. In any case, even if we assume that differences in disclosed asset allocations perfectly capture differences in risk, it is still true that those investors who are the most likely to be defaulted into TDFs - and to stay in TDFs - may be the least able to make an informed choice between TDFs and other investment vehicles.

\footnotetext{
${ }^{44}$ DOL: EBSA Federal Register: 29 CFR Part 2550, RIN 1210-AB38, October 20, 2010. On May 24, 2012, additional disclosure requirements were proposed, based "on evidence that plan participants and beneficiaries would benefit from additional information concerning these investments" (DOL: EBSA Federal Register: 29 CFR Part 2550, RIN 1210-AB38, May 24, 2012). In April 2013, “the Securities and Exchange Commission's Investor Advisory Committee recommended that the Commission develop a glide path illustration for target date funds that is based on a standardized measure of fund risk as a replacement for, or supplement to, an asset allocation glide path illustration." Between May 27, 2014 and July 3, 2014, the DOL reopened the public comment period. To the best of our knowledge, the rules are still pending.
} 


\section{Appendix}

\section{A.1 The Pension Protection Act of 2006}

\section{A.1.1 Overview}

The PPA of 2006 amends Title I of the Employee Retirement Income Security Act (ERISA) of 1974. Of particular interest to our study, it relieves sponsors of DC retirement plans of liability for investment losses when they default plan participants into "qualified default investment alternatives" (QDIAs). As specified by the Department of Labor's (DOL) Employee Benefits Security Administration (EBSA), QDIAs must be diversified to decrease probability of large losses; be managed by an investment manager/company registered under the Investment Company Act of 1940; not penalize or prevent a participant from transferring their assets from a QDIA to another investment alternative available under the plan; and not invest participant contributions directly in employer securities. ${ }^{45}$ Potential QDIAs include TDFs, BFs, and professionally managed accounts. Note that plan sponsors and fiduciaries are not relieved of liability for the prudent selection and monitoring of a QDIA.

\section{A.1.2 Timeline}

In January of 2005, a proposal regarding the funding of pensions was created, indicating new minimum funding requirements for pension plans with the hope of strengthening the overall pension system. Later that year, major pension reform bills were proposed in the House (The Pension Protection Act) and the Senate (The Pension Security and Transparency Act). The PPA of 2006 resulted from negotiations between the House and the Senate conducted in March of $2006 .{ }^{46}$ The final ruling was passed by the House on July 28, 2006, passed by the Senate on August 3, 2006, and

\footnotetext{
${ }^{45}$ DOL: EBSA Federal Register: 29 CFR Part 2550, October 24, 2007.

${ }^{46}$ Congressional Research Service Report for Congress, October 23, 2006.
} 
signed into law on August 17, 2006. On September 27, 2006, the DOL proposed rules regarding

"Default Investment Alternatives Under Participant Directed Individual Account Plans," listing TDFs among the set of QDIAs. These rules went into effect on December 24, 2007.

\section{A.2 Public Statements Summarizing Advantages and Disadvantages of TDFs}

Source for all quotes: DOL and SEC Joint Public Hearing on TDFs and Other Similar Investment Options: June 18, 2009.

Advantages:

- "Target date funds were expected to make investing easier for the typical American and avoid the need for investors to constantly monitor market movements and realign their personal investment allocations." SEC Chairman Mary Shapiro

- "Target Date Funds are one of the most important recent innovations in retirement savings. They provide a convenient way for an investor to purchase a mix of asset classes within a single fund that will rebalance the asset allocation and become more conservative as the investor ages." Karrie McMillan, general counsel of the Investment Company Institute

- "Target Date Fund investors avoid extreme asset allocations that we often observe in retirement savings." Karrie McMillan, general counsel of the Investment Company Institute

- "Target date funds were designed to be easy to use and require little maintenance." Richard Whitney, Director of Asset Allocation of T. Rowe Price

- "...the fundamental purpose of Target Date Funds is to provide investors a diversified, prudently-managed, appropriate exposure to investment risks." John Ameriks, economist and principal at the Vanguard Group 
- "When evaluating the performance of Target date funds, it's important to acknowledge the extreme severity of the financial meltdown we have just experienced ... in our view they performed as designed. In particular, in the vast majority of cases, older investors were exposed to far less risks than younger investors and consequently suffered less dramatic losses." John Ameriks, economist and principal at the Vanguard Group

- "... it is important for investors to stay committed to a retirement savings plan. Target Date Funds are designed to help participants maintain this discipline." Derrick Young, Chief Investment Officer of the Fidelity Global Asset Allocation Group

\section{Disadvantages:}

- "While Target Date Mutual Funds currently do a good job of describing their objectives, risks and glide paths, we do see gaps in the public understanding of target date funds." Karrie McMillan, general counsel of the Investment Company Institute

- "Retirees do a lot of different things with the money in these plans at the point of retirement, and so there is some debate around exactly how the money is going to be used ... it's very difficult to come up with a sort of specific answer that solves the problem for everybody." John Ameriks, economist and a principal at the Vanguard Group

- "We have serious concerns that these funds are fundamentally misleading to investors because they're allowed to be managed in ways that are inconsistent with reasonable expectations that are created by the titles and the use of the names." Marilyn Capelli-Dimitroff, Chair of the Certified Financial Planner Board of Standards

- "Appropriate disclosures are required and must be provided, but in reality, disclosures are seldom read or understood fully despite our ongoing education of clients." Marilyn CapelliDimitroff, Chair of the Certified Financial Planner Board of Standards 
- "When plan sponsors and participants started adopting TDFs in big meaningful numbers starting in 2002, the race was on for performance numbers, and this is where the train went off the track ... There is some theoretical rationale for employing a glide path through the accumulation phase. No credible rationale has ever been proffered for using a glide path in the distribution phase. This is what caused the unacceptably large losses in 2010 funds in 2008." Joe Nagengast, Target Date Analytics

- "... part of the concern here is when you have a fund of funds, it may become a lot easier to, for example, hide under-performing funds in Target Date Funds, [or] hide higher fee funds in a Target Date Fund that may not be completely appropriate." Dave Certner, Legislative Counselor and Legislative Policy Director at AARP

\section{A.3 Decomposing TDF variation}

In this section, we benchmark dispersion in TDF returns and risk-taking against both BFs and S\&P 500 index funds. In order to quantify the contribution of the cross-sectional dispersion on the overall dispersion of returns, we compute three measures. We describe the measures for TDFs, but they can just as easily be calculated for BFs and index funds. First, we compute the "Total Dispersion," the total standard deviation of returns for TDFs with a given target date. ${ }^{47}$ This is the variability of realized TDF returns around the overall average return for that target date, and measures the total risk faced by investors who invest in TDFs with target date $j$ : in a balanced panel, this variability can be thought of as the risk faced by an investor who is assigned randomly to a TDF at the beginning of the sample, and who stays in that TDF for the remainder of the

\footnotetext{
${ }^{47}$ For target date $j$ the Total Dispersion is defined as:

$$
\hat{\sigma}_{T j}=\sqrt{\frac{1}{\sum_{t=1}^{T_{j}} N_{j t}} \sum_{t=1}^{T_{j}} \sum_{i=1}^{N_{j t}}\left(r_{i j t}-\overline{\bar{r}}_{j}\right)^{2}},
$$

where $r_{i j t}$ is a TDF's yearly return and $\overline{\bar{r}}_{j}$ is the average return across all TDFs with target date $j$ and all years.
} 
sample. Second, we compute the "Market Dispersion," the standard deviation over time of the return on an equal-weighted portfolio of TDFs with a given target date. ${ }^{48}$ Third, we compute the "Fund Dispersion," the standard deviation within a given target date. ${ }^{49}$ In a balanced panel, this is the extra risk that an investor bears because of having chosen the $i$-th TDF with target date $j$, as opposed to an equal-weighted portfolio of TDFs with target date $j$. This general approach can also be used to decompose the dispersion of alphas, idiosyncratic volatilities, betas, and $R^{2}$ s. Results are presented in Table A.1.

We first focus on the variability of realized TDF returns. Looking across the five samples of TDFs, we see that much of the risk associated with investing in TDFs comes from Market Dispersion: Total Dispersion ranges between $14.0 \%$ and 18.9\%, and Market Dispersion ranges between $13.6 \%$ and $18.7 \%$. However, consistent with our earlier findings, there remains significant Fund Dispersion. Fund Dispersion ranges from 2.4\% for $2035-2040$ and $2045-2050$ funds, to $3.2 \%$ for 2005-2010 funds, confirming that there is more Fund Dispersion in realized TDF returns when target dates are near than when they are far.

For BFs, which arguably have more discretion over asset allocation, market timing, and security selection, Total Dispersion is $13.9 \%$ and Fund Dispersion is $5.1 \%$. In contrast, for S\&P 500 index funds, Total Dispersion is $17.9 \%$ and Fund Dispersion is only $0.5 \%$. Hence, all five target dates expose investors to greater Total Dispersion but less Fund Dispersion than traditional BFs. Perhaps more surprisingly, 2035-2045 TDFs expose investors to greater Total Dispersion than

${ }^{48}$ Market Dispersion is defined as:

$$
\hat{\sigma}_{M j}=\sqrt{\frac{1}{\sum_{t=1}^{T_{j}} N_{j t}} \sum_{t=1}^{T_{j}} N_{j t}\left(\bar{r}_{j t}-\overline{\bar{r}}_{j}\right)^{2}},
$$

where $\bar{r}_{j t}$ is the year- $t$ return on an equal-weighted portfolio of TDFs with target date $j$.

${ }^{49}$ Fund Dispersion is defined as:

$$
\sqrt{\hat{\sigma}_{T j}^{2}-\hat{\sigma}_{M j}^{2}}=\sqrt{\frac{1}{\sum_{t=1}^{T_{j}} N_{j t}} \sum_{t=1}^{T_{j}} \sum_{i=1}^{N_{j t}}\left(r_{i j t}-\bar{r}_{j t}\right)^{2} .}
$$


S\&P 500 index funds, which invest close to $100 \%$ in U.S. equity. The patterns are similar when we switch our focus from total returns to idiosyncratic returns (measured using the annualized five-factor alphas from Table 3). On average, Fund Dispersion in idiosyncratic returns explains approximately $70 \%$ of the Fund Dispersion in total returns.

When we turn to idiosyncratic volatility, we find that Fund Dispersion always exceeds Market Dispersion, but by a modest amount. The differences between Fund Dispersion and Market Dispersion are more pronounced for five-factor model $R^{2} \mathrm{~s}$ and U.S. equity betas. For $R^{2} \mathrm{~s}$, Total Dispersion ranges between 3.5\% and 4.4\%, and Market Dispersion ranges between $0.5 \%$ and $1.0 \%$. Overall, Table A.1 reveals that TDFs with the same target date expose investors to significantly different levels of idiosyncratic and systematic risk. With respect to economic significance, the dispersion within each sample of TDFs is about half as large as within the full sample of BFs. 


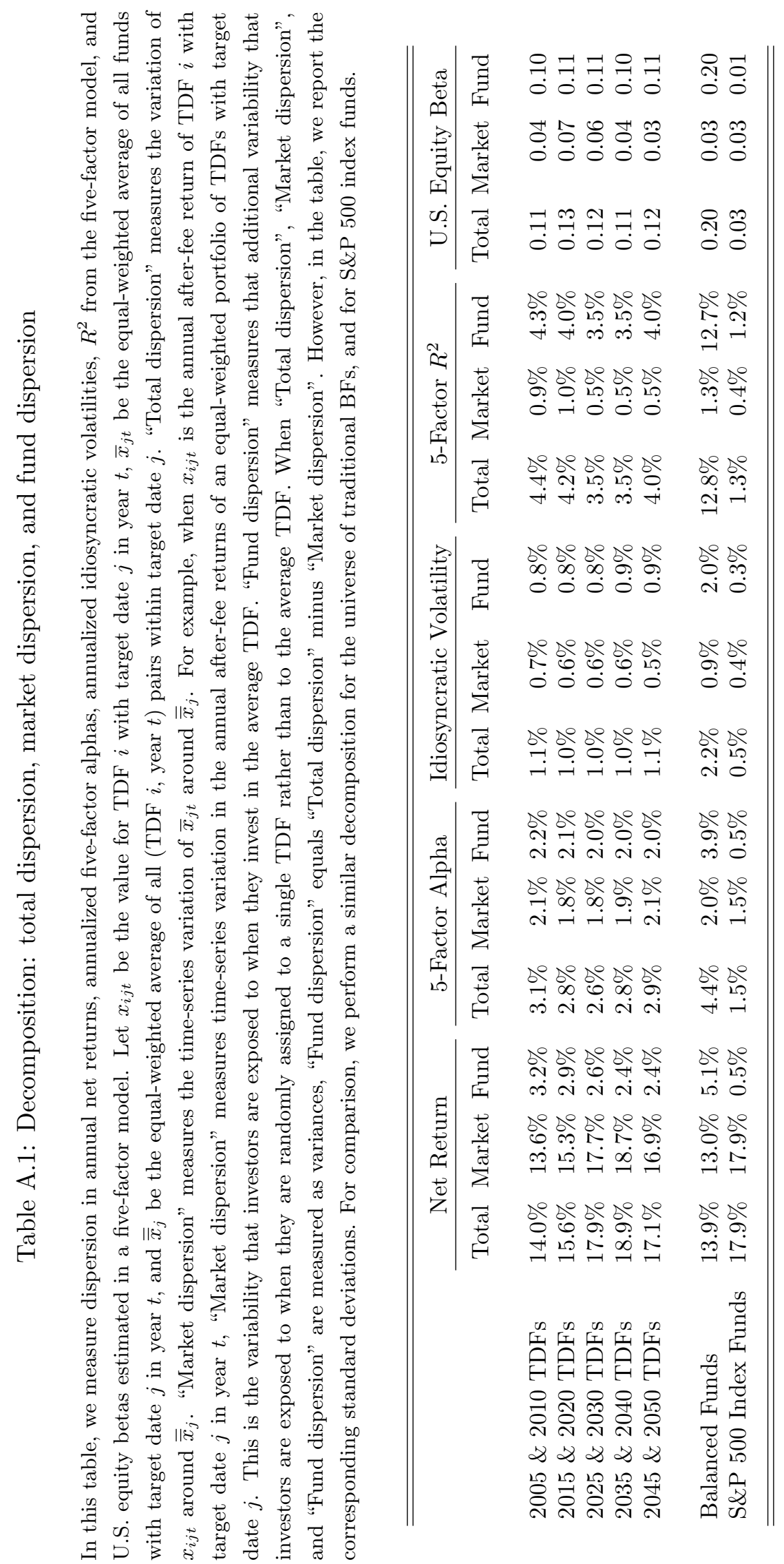


Table A.2: Number of mutual fund families and TDFs based on market share and whether they entered post PPA

The top panel reports the number of mutual fund families that offer TDFs each year, based on their share in the TDF market (low, medium, or high), and on whether they entered the TDF market before or after December 31,2006 (pre-PPA versus post-PPA). The bottom panel reports the corresponding number of TDF-month observations. Note that the total number of TDF-month observations exceeds those included in the regressions in Tables 8-10 because we do not require that we possess sufficient historical return data to estimate five-factor alphas.

\begin{tabular}{|c|c|c|c|c|c|c|c|}
\hline & \multicolumn{7}{|c|}{ Number of Families } \\
\hline & \multicolumn{3}{|c|}{ Pre-PPA Family } & \multicolumn{3}{|c|}{ Post-PPA Family } & \multirow[t]{2}{*}{ All } \\
\hline & Low & Medium & High & Low & Medium & High & \\
\hline 2000 & 1 & 0 & 3 & 0 & 0 & 0 & 4 \\
\hline 2001 & 2 & 0 & 3 & 0 & 0 & 0 & 5 \\
\hline 2002 & 2 & 2 & 2 & 0 & 0 & 0 & 6 \\
\hline 2003 & 4 & 4 & 1 & 0 & 0 & 0 & 9 \\
\hline 2004 & 5 & 5 & 3 & 0 & 0 & 0 & 13 \\
\hline 2005 & 12 & 4 & 4 & 0 & 0 & 0 & 20 \\
\hline 2006 & 16 & 5 & 4 & 0 & 0 & 0 & 25 \\
\hline 2007 & 16 & 5 & 4 & 8 & 0 & 0 & 33 \\
\hline 2008 & 17 & 5 & 4 & 17 & 1 & 0 & 44 \\
\hline 2009 & 15 & 5 & 4 & 15 & 1 & 0 & 40 \\
\hline 2010 & 14 & 6 & 4 & 14 & 1 & 0 & 39 \\
\hline 2011 & 13 & 8 & 3 & 15 & 1 & 0 & 40 \\
\hline 2012 & 11 & 8 & 3 & 14 & 1 & 0 & 37 \\
\hline
\end{tabular}

\begin{tabular}{|c|c|c|c|c|c|c|c|}
\hline & \multicolumn{7}{|c|}{ Number of TDFs } \\
\hline & \multicolumn{3}{|c|}{ Pre-PPA Family } & \multicolumn{3}{|c|}{ Post-PPA Family } & \multirow[t]{2}{*}{ All } \\
\hline & Low & Medium & High & Low & Medium & High & \\
\hline 2000 & 60 & 0 & 185 & 0 & 0 & 0 & 245 \\
\hline 2001 & 115 & 0 & 192 & 0 & 0 & 0 & 307 \\
\hline 2002 & 133 & 64 & 152 & 0 & 0 & 0 & 349 \\
\hline 2003 & 165 & 207 & 99 & 0 & 0 & 0 & 471 \\
\hline 2004 & 190 & 384 & 192 & 0 & 0 & 0 & 766 \\
\hline 2005 & 575 & 394 & 315 & 0 & 0 & 0 & 1284 \\
\hline 2006 & 1114 & 251 & 421 & 0 & 0 & 0 & 1786 \\
\hline 2007 & 1335 & 418 & 468 & 436 & 0 & 0 & 2657 \\
\hline 2008 & 1560 & 488 & 547 & 969 & 99 & 0 & 3663 \\
\hline 2009 & 1471 & 704 & 588 & 1433 & 108 & 0 & 4304 \\
\hline 2010 & 1411 & 926 & 701 & 1154 & 119 & 0 & 4311 \\
\hline 2011 & 1229 & 1376 & 612 & 1154 & 120 & 0 & 4491 \\
\hline 2012 & 1247 & 1555 & 611 & 1304 & 120 & 0 & 4837 \\
\hline
\end{tabular}




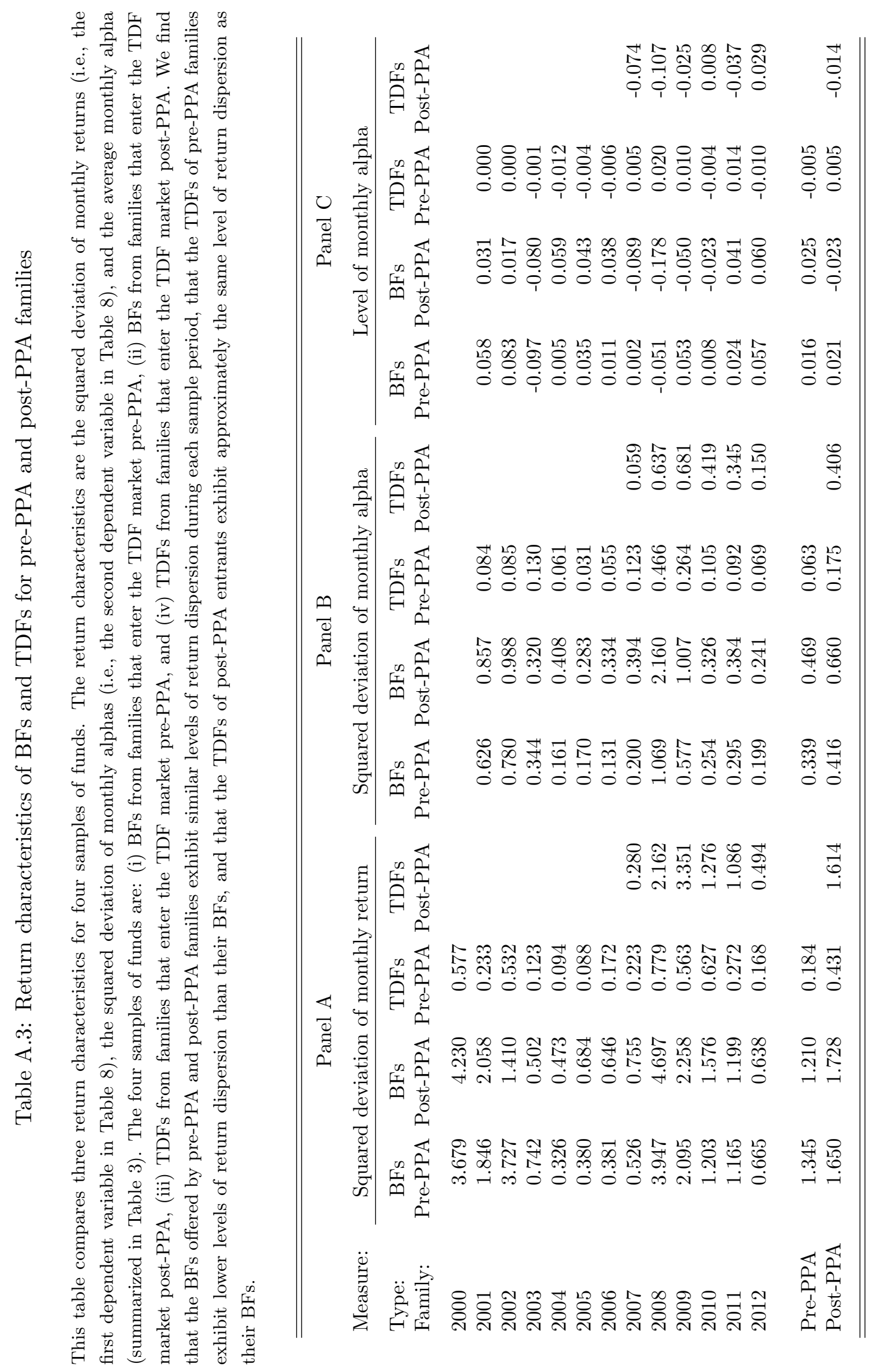




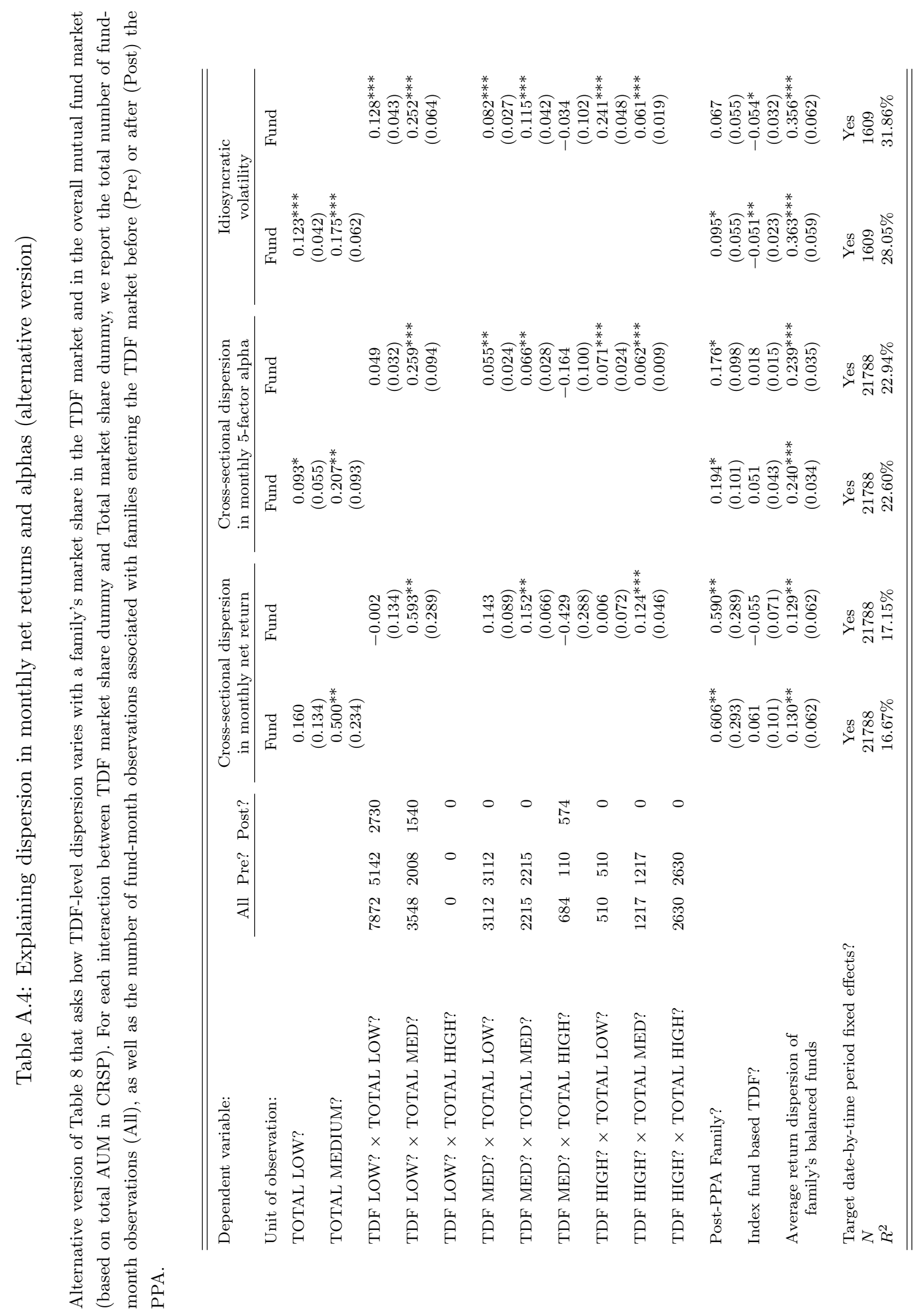




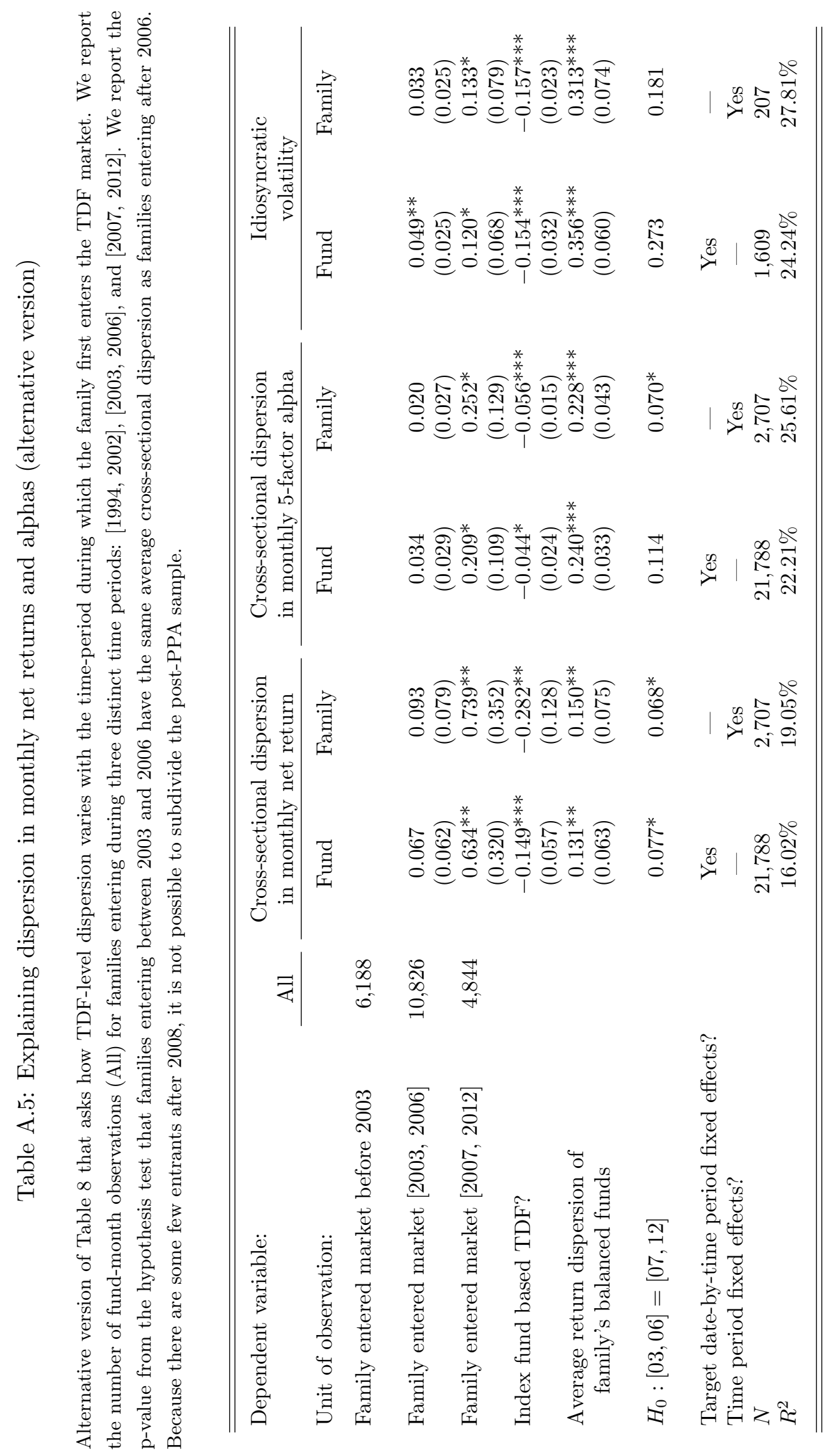




\section{References}

[1] Agnew, Julie R., Lisa R. Szykman, Stephen P. Utkus, and Jean A. Young. 2012. "Target-Date Funds: Survey and Administrative Evidence." Working Paper.

[2] Agnew, Julie, Pierluigi Balduzzi, and Annika Sundén. 2003. "Portfolio Choice and Trading in a Large 401(k) Plan." American Economic Review 93, 193-215.

[3] Ameriks, John, Dean J. Hamilton, and Liqian Ren. 2011. "Investor Comprehension and Usage of Target-Date Funds: 2010 Survey." Vanguard Investment Counseling and Research. January 2011.

[4] Amihud, Yakov and Ruslan Goyenko. 2013. "Mutual Fund's $R^{2}$ as Predictor of Performance." Review of Financial Studies 26, 667-694.

[5] Balduzzi, Pierluigi, and Anthony W. Lynch. 1999. "Transaction Costs and Predictability: Some Utility Cost Calculations." Journal of Financial Economics 52, 47-78.

[6] Benartzi, Shlomo, and Richard H. Thaler. 2001. "Naive Diversification Strategies in Defined Contribution Saving Plans." American Economic Review 91, 79-98.

[7] Benzoni, Luca, Pierre Collin-Dufresne, and Robert S. Goldstein. 2007. "Portfolio Choice over the Life-Cycle when the Stock and Labor Markets Are Cointegrated." Journal of Finance 62, 2123-2168.

[8] Berk, Jonathan, Richard Stanton, and Josef Zechner. 2010. "Human Capital, Bankruptcy, and Capital Structure." Journal of Finance 65, 891-926.

[9] Bodie, Zvi, Robert C. Merton, and William F. Samuelson. 1992. "Labor Supply Flexibility and Portfolio Choice in a Life Cycle Model." Journal of Economic Dynamics and Control 16, $427-49$.

[10] Brown, Keith C., Harlow, W. V., and Laura T. Starks. 1996. "Of Tournaments and Temptations: An Analysis of Managerial Incentives in the Mutual Fund Industry." Journal of Finance $51,85-110$.

[11] Carhart, Mark M. 1997. "On Persistence in Mutual Fund Performance." Journal of Finance $52,57-82$.

[12] Chalmers, John, and Jonathan Reuter. 2014. "What is the Impact of Financial Advisors on Retirement Portfolio Choices and Outcomes?" Working Paper.

[13] Cocco, João F., Francisco J. Gomes, and Pascal J. Maenhout. 2005. "Consumption and Portfolio Choice over the Life Cycle." Review of Financial Studies 18, 491-533.

[14] Del Guercio, Diane, and Paula Tkac. 2002. "The Determinants of the Flow of Funds of Managed Portfolios: Mutual Funds vs. Pension Funds." Journal of Financial and Quantitative Analysis, $37,523-557$.

[15] Del Guercio, Diane, and Jonathan Reuter. 2014. "Mutual Fund Performance and the Incentive to Generate Alpha." Journal of Finance, 69, 1673-1704. 
[16] Elton, Edwin J., Martin J. Gruber, Andre de Souza, and Christopher R. Blake. 2014. "Target Date Funds: Characteristics and Performance." 2015. Forthcoming, Review of Asset Pricing Studies.

[17] Evans, Richard B. 2010. "Mutual Fund Incubation." Journal of Finance 65, 1581-1611.

[18] Gomes, Francisco J., Laurence J. Kotlikoff, and Luis M. Viceira. 2008. "Optimal Lifecycle Investing with Flexible Labor Supply: A Welfare Analysis of Lifecycle Funds." American Economic Review 98, 297-303.

[19] Jagannathan, Ravi, and Narayana R. Kocherlakota. 1996. "Why Should Older People Invest Less in Stocks Than Younger People?" Quarterly Review, Federal Reserve Bank of Minneapolis, Summer, 11-23.

[20] Lynch, Anthony W. 2001. "Portfolio Choice and Equity Characteristics: Characterizing the Hedging Demands Induced by Return Predictability." Journal of Financial Economics 62, $67-130$.

[21] Madrian, Brigitte C., and Dennis F. Shea. 2001. "The Power of Suggestion: Inertia in 401(k) Participation and Savings Behavior." Quarterly Journal of Economics 116, 1149-1187.

[22] Merton, Robert C. 1971. "Optimum Consumption and Portfolio Rules in a Continuous-Time Model." Journal of Economic Theory 3, 373-413.

[23] Mitchell, Olivia S., Gary R. Mottola, Stephen P. Utkus, and Takeshi Yamaguchi. 2009. "Default, Framing and Spillover Effects: The Case of Lifecycle Funds in 401(k) Plans." Working Paper.

[24] Mitchell, Olivia S., and Stephen P. Utkus. 2012. "Target-Date Funds in 401(k) Retirement Plans." Working Paper.

[25] Morrin, Maureen, Susan M. Broniarczyk, and J. Jeffrey Inman. 2012. "Plan Format and Participation in 401(k) Plans: The Moderating Role of Investor Knowledge." Journal of Public Policy and Marketing 31, 254-268.

[26] Pagliaro, Cynthia A., and Stephen P. Utkus. 2010. "Mixed Target-Date Investors in Defined Contribution Plans." Vanguard Center for Retirement Research, September 2010.

[27] Pang, Gaobo, and Mark J. Warshawsky. 2009. "Asset Allocations and Risk-Return Tradeoffs of Target-Date Funds." Working Paper.

[28] Park, Youngkyun. 2009. "Investment Behavior of Target-Date Fund Users Having Other Funds in 401(k) Plan Accounts." EBRI Notes 30.

[29] Park, Youngkyun, and Jack L. VanDerhei. 2008. "401(k) Plan Participant Investments in Lifecycle Funds under Plan Sponsors Initiative." Working Paper.

[30] Pástor, Ľuboš, and Robert F. Stambaugh. 2011. "Are Stocks Really Less Volatile in the Long Run?" Journal of Finance 67, 431-477.

[31] Pool, Veronika K., Clemens Sialm, and Irina Stefanescu. 2013. "It Pays to Set the Menu: Mutual Fund Investment Options in 401(k) Plans." Working Paper.

[32] Sandhya, Vallapuzha V. 2011. "Agency Problems in Target-Date Funds.” Working Paper. 
[33] Shiller, Robert J. 2005. "The Life-Cycle Personal Accounts Proposal for Social Security: An Evaluation." Working Paper.

[34] Sialm, Clemens, Laura Starks, and Hanjiang Zhang. 2015. "Defined Contribution Pension Plans: Sticky or Discerning Money?" Journal of Finance 70, 805-838.

[35] Special Committee on Aging. 2009. "Target Date Retirement Funds: Lack of Clarity Among Structures and Fees Raises Concerns." U.S. Senate Summary of Committee Research.

[36] Viceira, Luis M. 2009. Lifecycle Funds. In Overcoming the Saving Slump: How to Increase the Effectiveness of Financial Education and Saving Programs. Annamaria Lusardi ed. University of Chicago Press.

[37] Yamaguchi, Takeshi, Olivia S. Mitchell, Gary R. Mottola, and Steven P. Utkus. 2007. "Winners and Losers: 401(k) Trading and Portfolio Performance." Working Paper. 


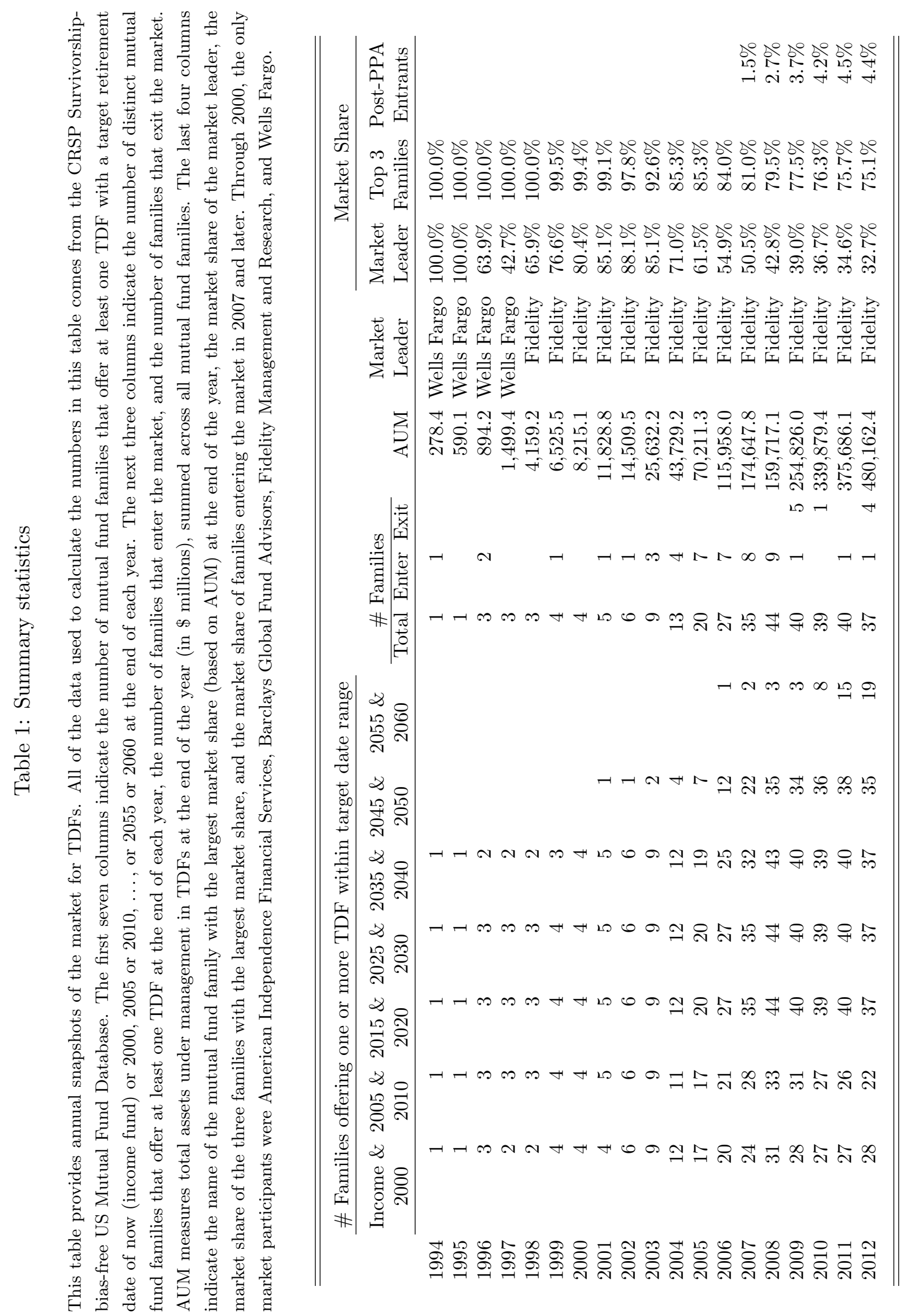




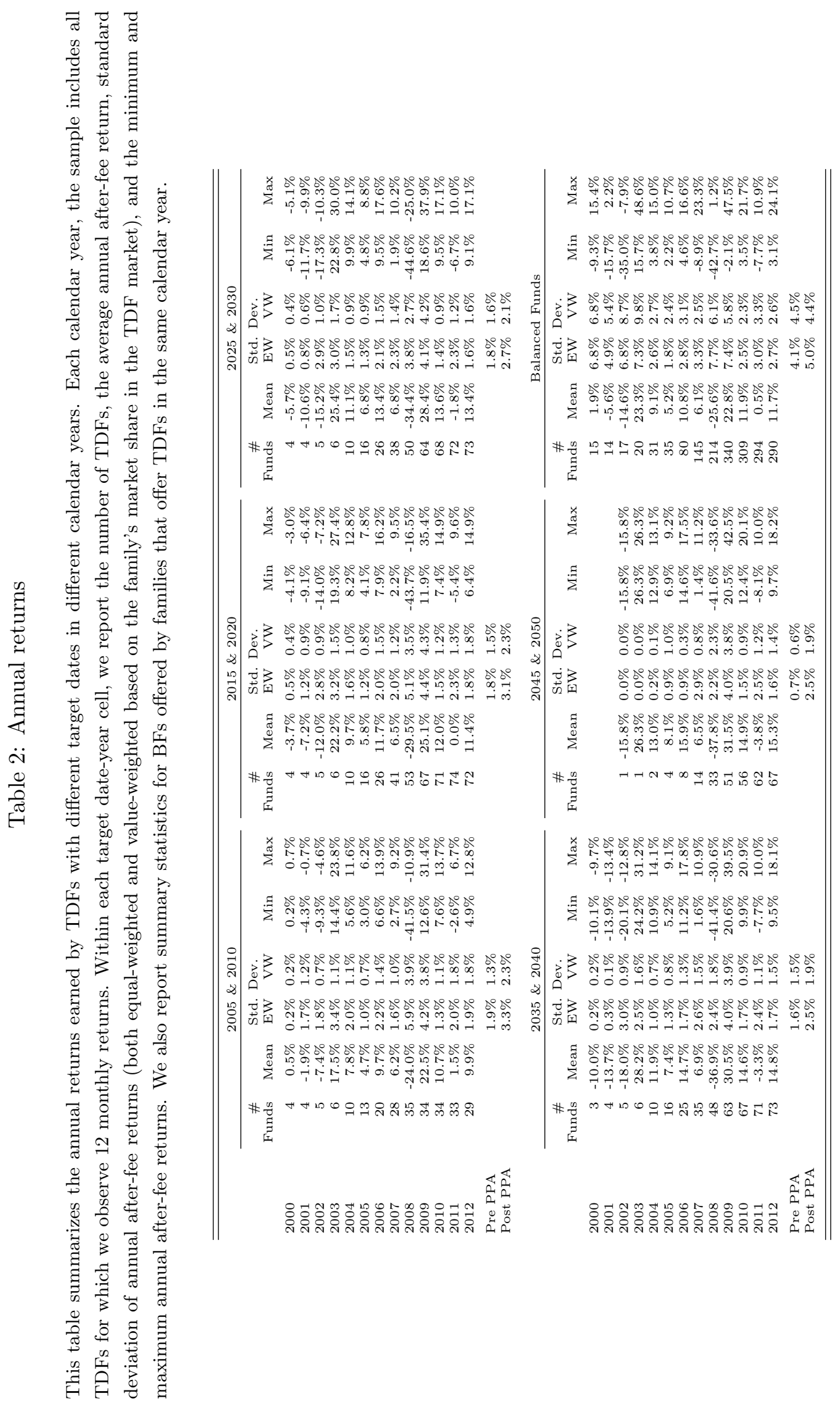




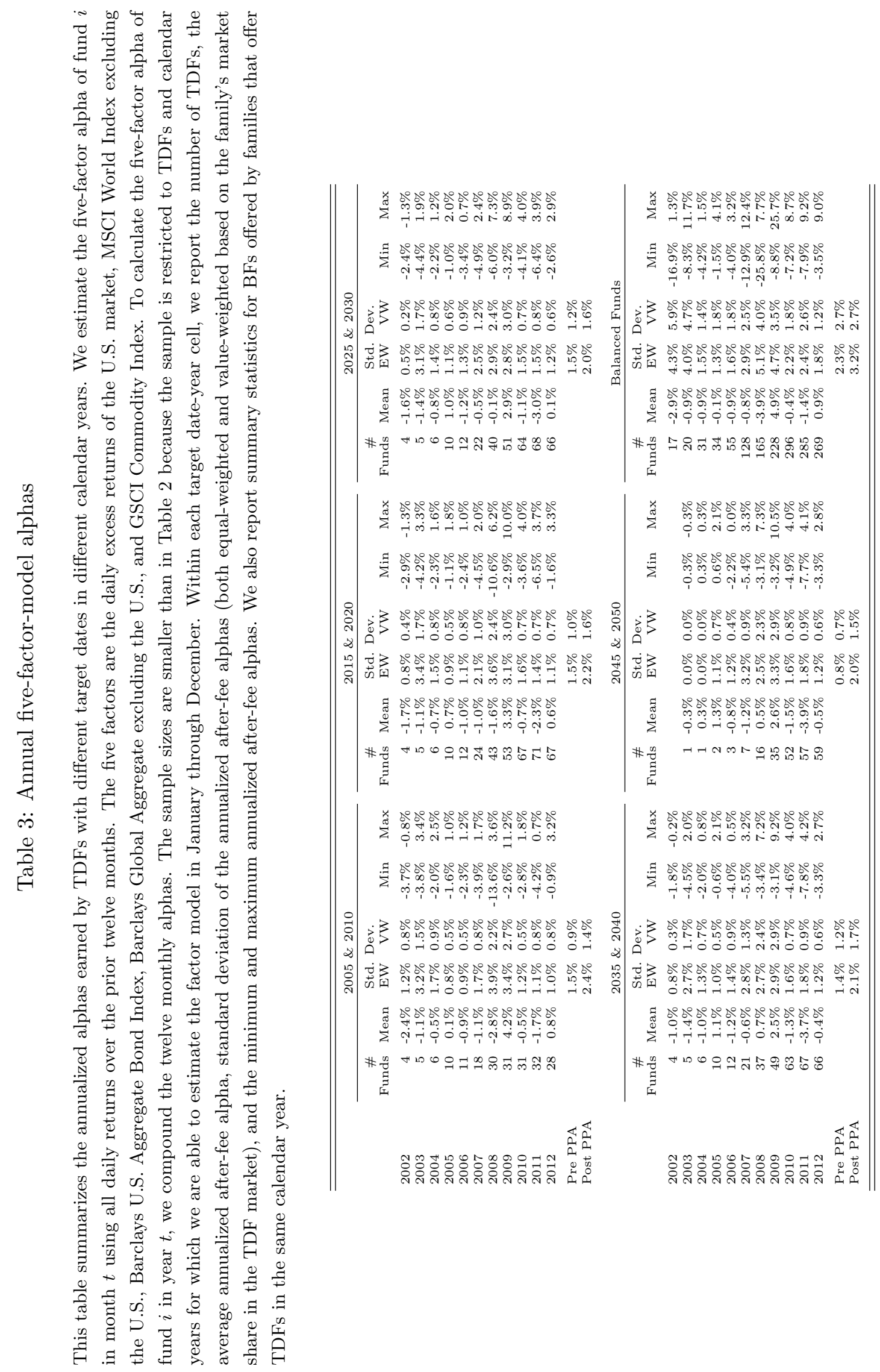




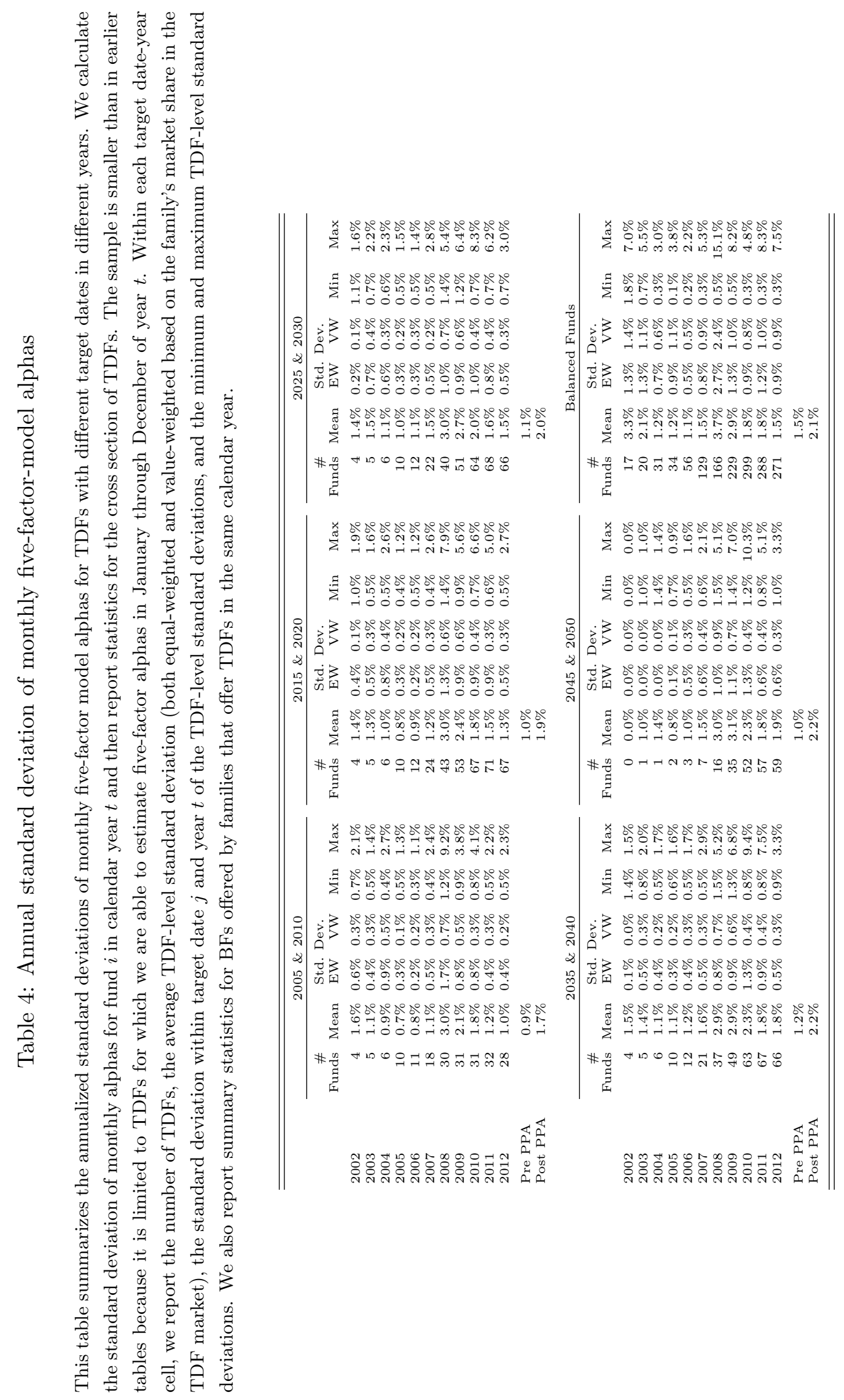




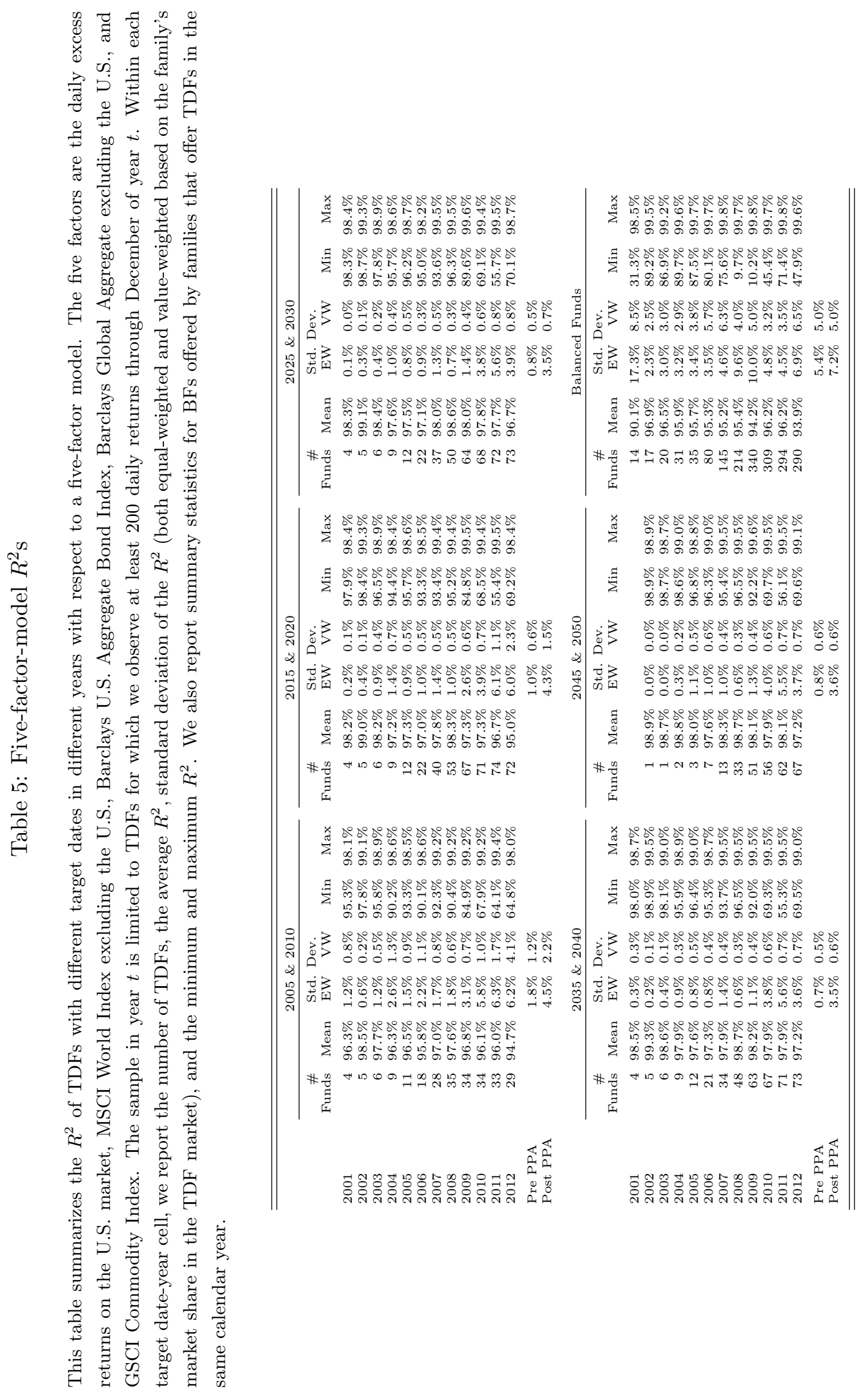




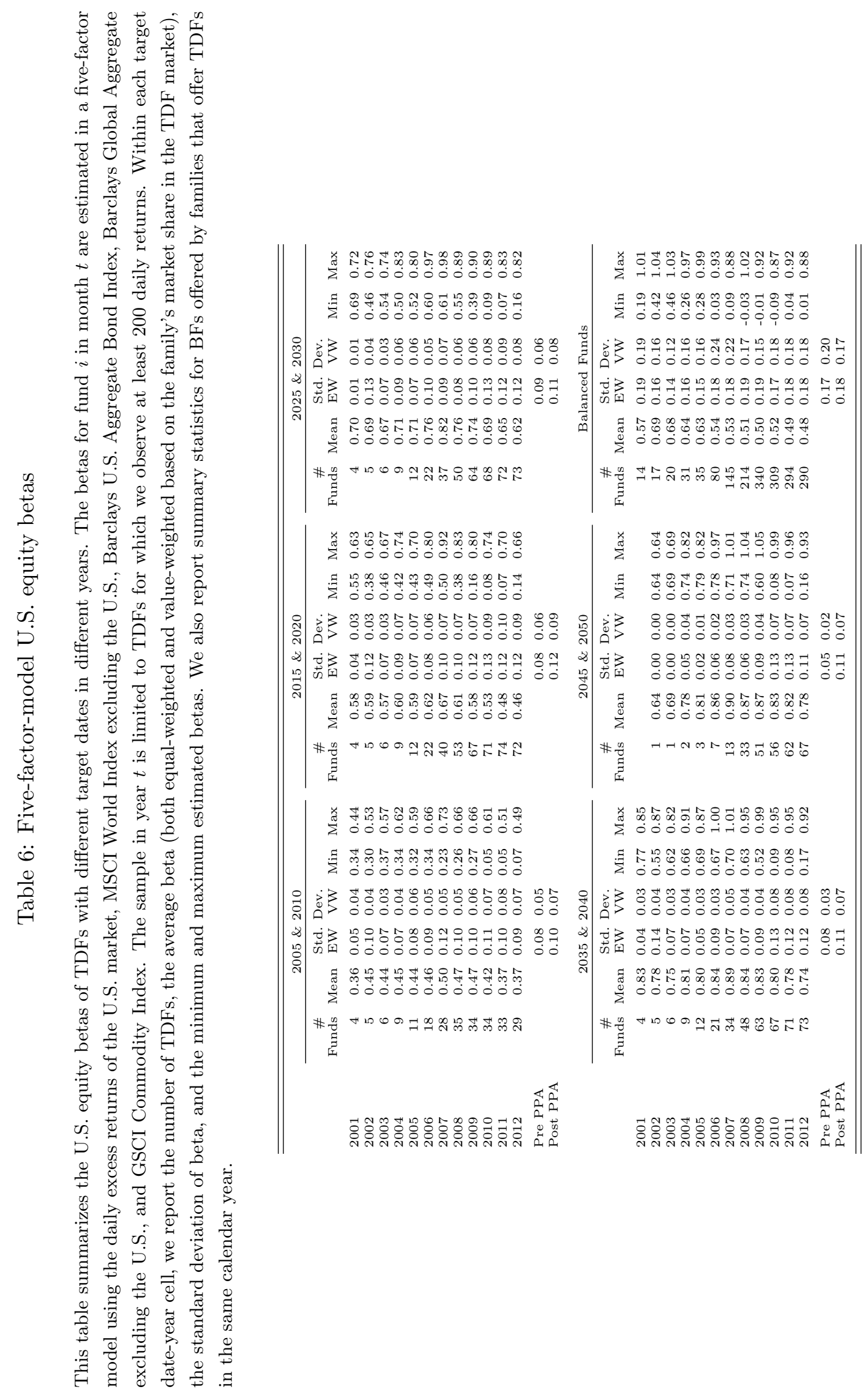


Table 7: Flows and performance

The unit of observation is the TDF offered by family $i$ with target date $j$. The dependent variable is estimated percentage net flow, measured over the 12 months ending in December of year $t$. The full set of independent variables includes: the lagged net return, measured over the 12 months ending in December of year $t-1$; the lagged five-factor alpha, measured over the same 12-month period; dummy variables that equal one if the lagged five-factor-alpha are in the first, second, third, or fourth quartiles of the distribution for target date $j$ in year $t-1$; the standard deviation of lagged monthly flows in year $t-1$; the lagged net flow in year $t-1$; the natural logarithm of the number of funds with target date $j$ in December of year $t$; a dummy equal to one if the fund was introduced after December 2006; a dummy equal to one if the fund was offered by a family that entered the TDF market after December 2006; the fund-level expense ratio measured in year $t$ (reported by CRSP); the natural logarithm of the fund assets in December of year $t-1$; and the natural logarithm of the family assets in December of year $t-1$. We control for both year fixed effects and target-date fixed effects. The sample in the first four columns includes all TDFs with target dates between 2005 and 2050 for which we observe the dependent and independent variables. The sample in the fifth column includes all BFs offered by families that simultaneously offer at least one TDF in year $t$. Estimation is via OLS. Standard errors are simultaneously clustered on family and year. ${ }^{*},{ }^{* *}$, and ${ }^{* * *}$ denote statistical significance at the $10 \%$ level, $5 \%$ level, and $1 \%$ level, respectively.

\begin{tabular}{|c|c|c|c|c|c|}
\hline \multirow{3}{*}{$\begin{array}{l}\text { Dependent variable: } \\
\text { Sample: } \\
\text { Net return, year } t-1\end{array}$} & \multicolumn{5}{|c|}{ Net flow, year $t$} \\
\hline & \multicolumn{4}{|c|}{ TDFs } & \multirow{2}{*}{$\begin{array}{c}\text { Balanced } \\
0.505^{* * *} \\
(0.159)\end{array}$} \\
\hline & $\begin{array}{c}0.361 \\
(0.420)\end{array}$ & $\begin{array}{c}0.159 \\
(0.267)\end{array}$ & $\begin{array}{c}0.240 \\
(0.242)\end{array}$ & & \\
\hline 5 -factor alpha, year $t-1$ & $\begin{array}{l}2.430^{* * *} \\
(0.914)\end{array}$ & $\begin{array}{l}2.009^{* *} \\
(0.844)\end{array}$ & $\begin{array}{l}1.807^{* * *} \\
(0.639)\end{array}$ & & $\begin{array}{c}0.905^{*} \\
(0.472)\end{array}$ \\
\hline 5 -factor alpha in fourth quartile? & & & & $\begin{array}{c}0.070^{*} \\
(0.042)\end{array}$ & \\
\hline 5 -factor alpha in third quartile? & & & & $\begin{array}{c}0.010 \\
(0.034)\end{array}$ & \\
\hline 5-factor alpha in second quartile? & & & & - & \\
\hline 5-factor alpha in first quartile? & & & & $\begin{array}{l}-0.079 * * * \\
(0.029)\end{array}$ & \\
\hline $\begin{array}{l}\text { Volatility of monthly net } \\
\text { returns, year } t-1\end{array}$ & & $\begin{array}{r}-8.616^{*} \\
(4.584)\end{array}$ & $\begin{array}{l}-11.467 * * * \\
(2.804)\end{array}$ & $\begin{array}{l}-11.666 * * * \\
(2.717)\end{array}$ & $\begin{array}{l}-1.036 \\
(1.150)\end{array}$ \\
\hline Net flow, year $t-1$ & & & $\begin{array}{l}0.308^{* * *} \\
(0.019)\end{array}$ & $\begin{array}{l}0.304^{* * *} \\
(0.019)\end{array}$ & $\begin{array}{l}0.447^{* * *} \\
(0.039)\end{array}$ \\
\hline $\begin{array}{l}\text { Ln number of funds with } \\
\text { target date } j \text { in year } t\end{array}$ & & $\begin{array}{l}0.103^{* * *} \\
(0.034)\end{array}$ & $\begin{array}{c}0.052 \\
(0.079)\end{array}$ & $\begin{array}{c}0.040 \\
(0.070)\end{array}$ & \\
\hline Fund introduced after $2006 ?$ & & $\begin{array}{l}0.335^{* * *} \\
(0.066)\end{array}$ & $\begin{array}{c}0.082 \\
(0.058)\end{array}$ & $\begin{array}{c}0.075 \\
(0.060)\end{array}$ & $\begin{array}{c}0.114 \\
(0.177)\end{array}$ \\
\hline $\begin{array}{l}\text { Fund managed by family entering } \\
\text { TDF market after } 2006 ?\end{array}$ & & $\begin{array}{l}-0.184^{* *} \\
(0.090)\end{array}$ & $\begin{array}{c}-0.045 \\
(0.058)\end{array}$ & $\begin{array}{c}-0.037 \\
(0.063)\end{array}$ & $\begin{array}{c}-0.002 \\
(0.024)\end{array}$ \\
\hline Expense ratio, year $t$ & & $\begin{array}{c}-0.058 \\
(0.038)\end{array}$ & $\begin{array}{c}-0.017 \\
(0.026)\end{array}$ & $\begin{array}{c}-0.011 \\
(0.026)\end{array}$ & $\begin{array}{c}-0.002 \\
(0.012)\end{array}$ \\
\hline Ln fund size, year $t-1$ & & $\begin{array}{c}0.000 \\
(0.021)\end{array}$ & $\begin{array}{c}0.005 \\
(0.011)\end{array}$ & $\begin{array}{c}0.002 \\
(0.011)\end{array}$ & $\begin{array}{r}-0.009^{*} \\
(0.005)\end{array}$ \\
\hline Ln family size, year $t-1$ & & $\begin{array}{c}0.027 \\
(0.024)\end{array}$ & $\begin{array}{c}0.007 \\
(0.013)\end{array}$ & $\begin{array}{c}0.008 \\
(0.013)\end{array}$ & $\begin{array}{c}0.002 \\
(0.009)\end{array}$ \\
\hline$H_{0}:$ Net return $=5$-factor alpha? & $0.001^{* * *}$ & $0.002^{* * *}$ & $0.015^{* *}$ & & 0.500 \\
\hline Calendar year fixed effects? & Yes & Yes & Yes & Yes & Yes \\
\hline Target date fixed effects? & Yes & Yes & Yes & Yes & Yes \\
\hline$N$ & 1,285 & 1,105 & 1,076 & 1,076 & 1,158 \\
\hline$R^{2}$ & $15.04 \%$ & $25.13 \%$ & $51.09 \%$ & $51.38 \%$ & $38.67 \%$ \\
\hline
\end{tabular}




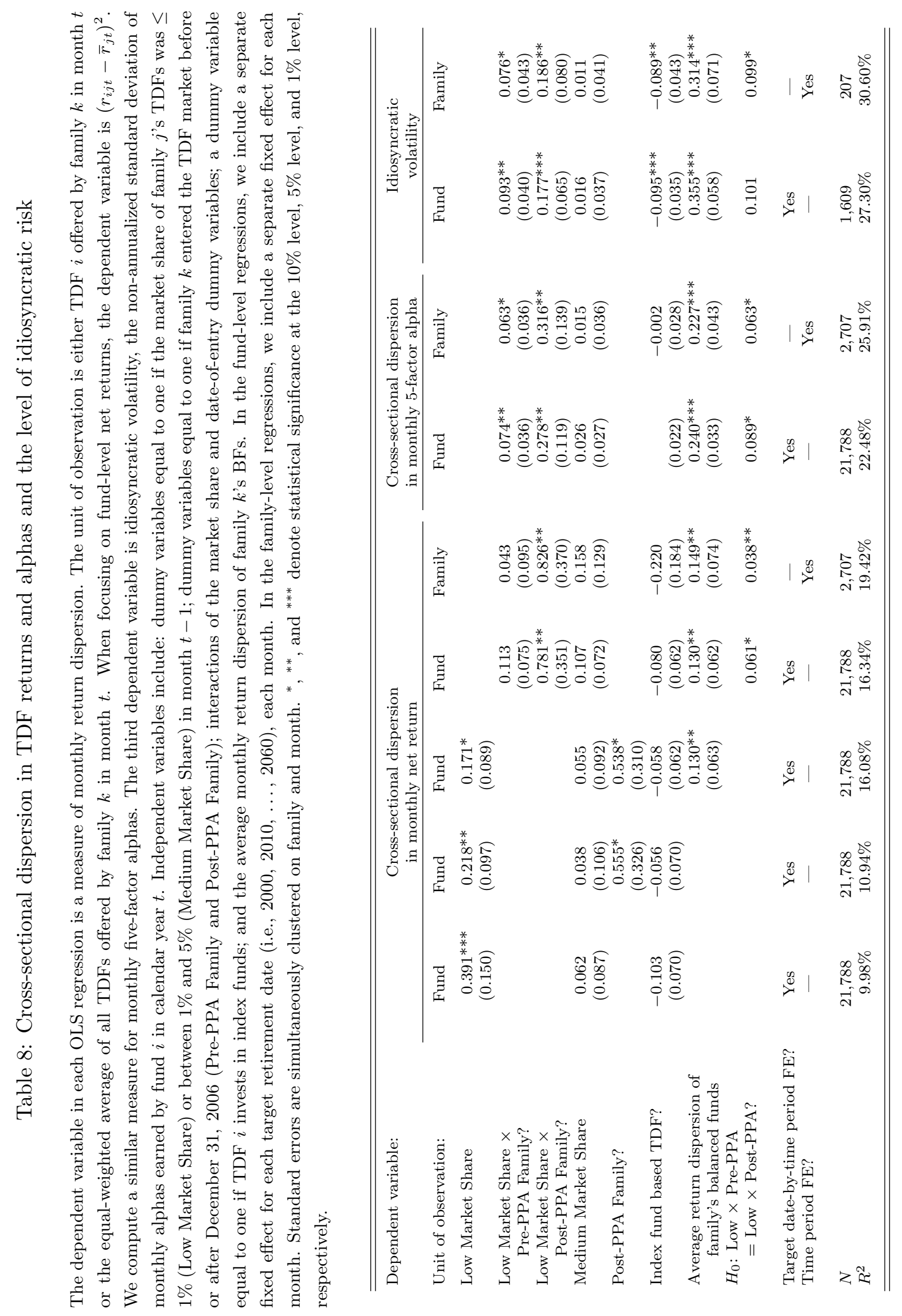




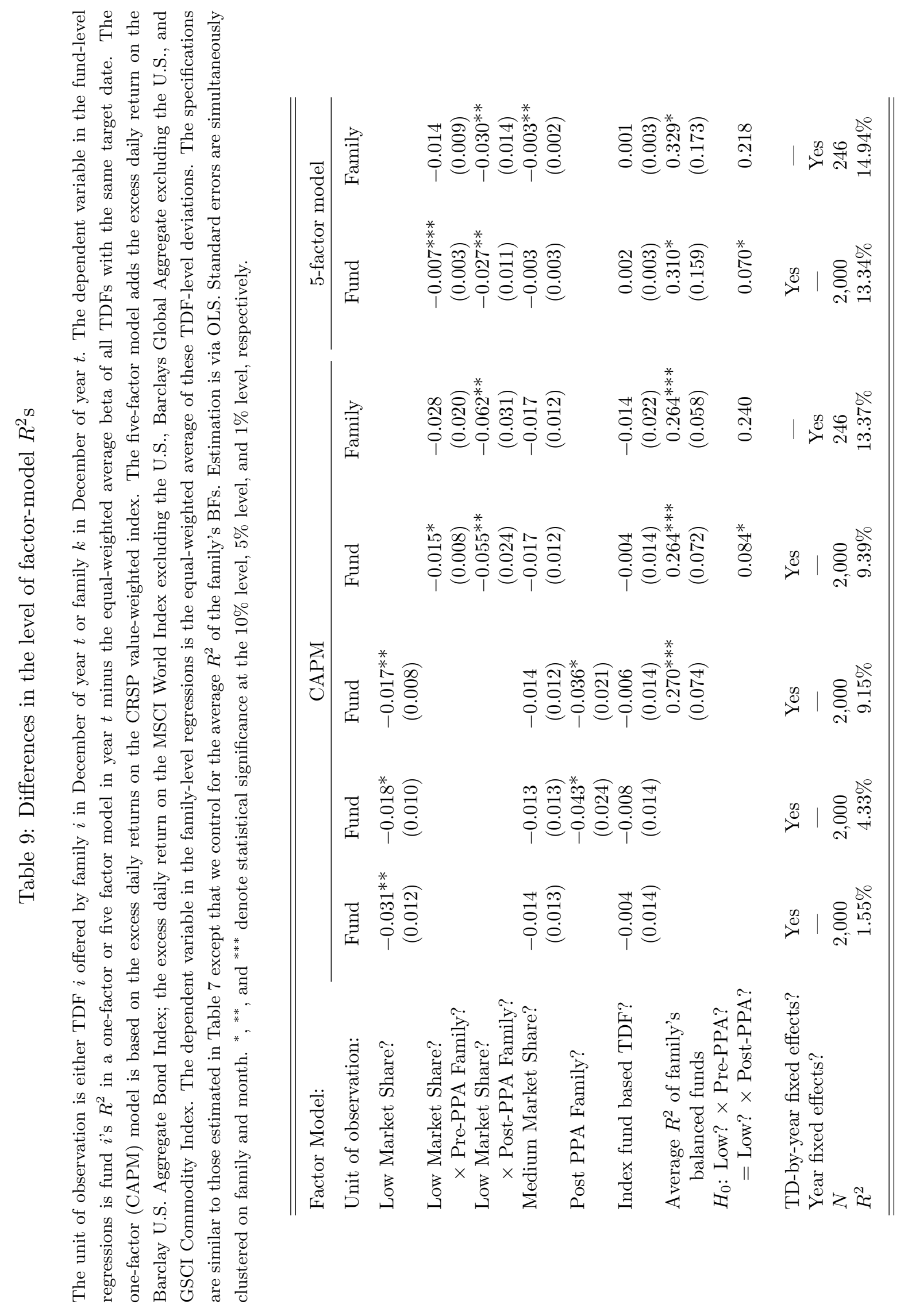




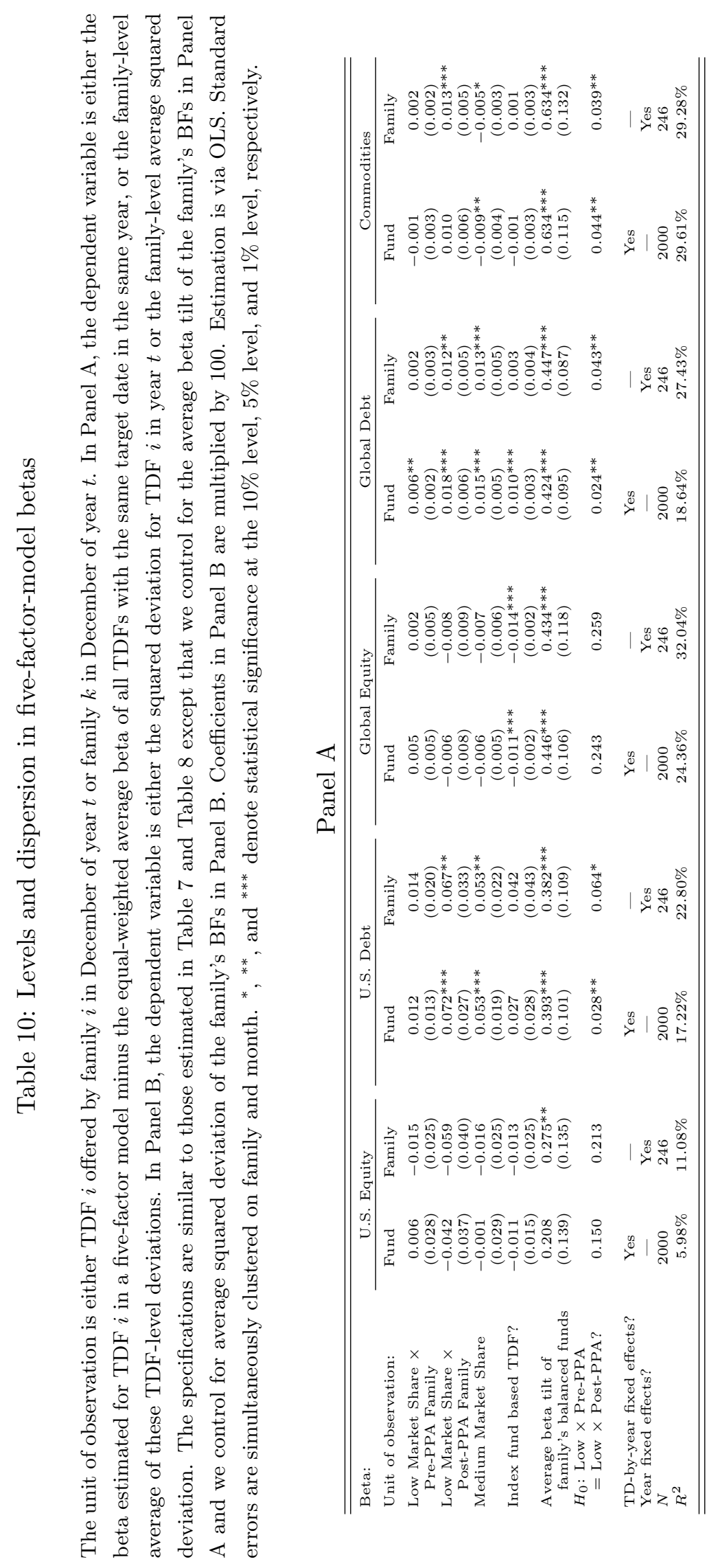




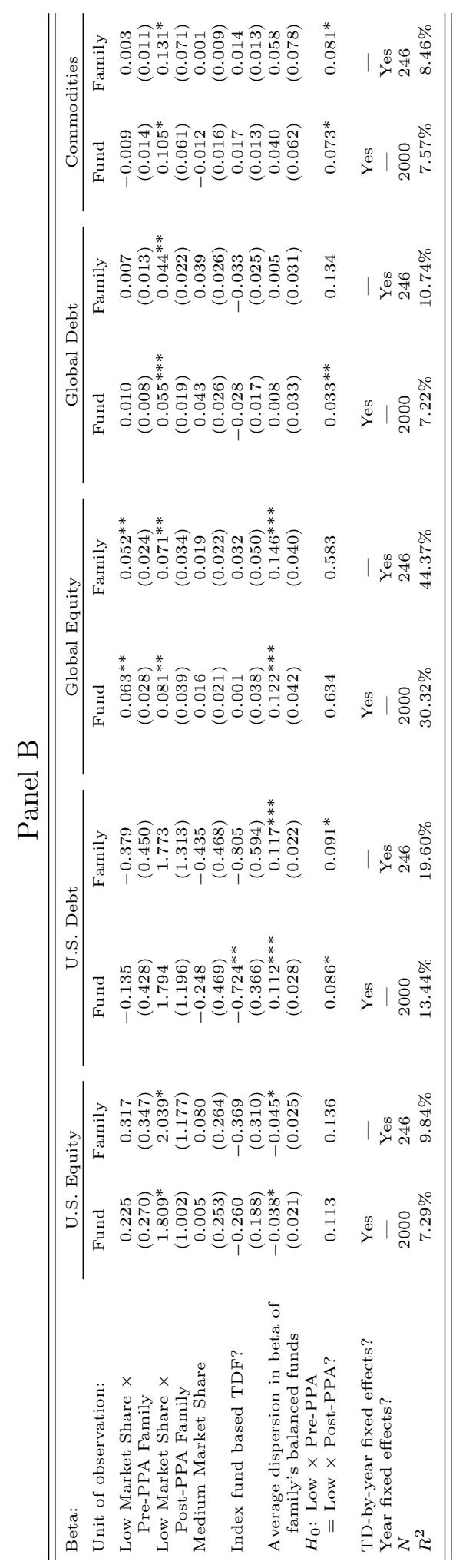


Table 11: BrightScope sample: summary statistics

We obtained data on 16,766 investment menus from BrightScope, Inc. The unit of observation is retirement plan $i$ offered by firm $j$ in industry $k$ in 2010. The sample is limited to single-employer 401(k) and 403(b) retirement plans. Plan-level characteristics include assets under management (across all investment options), the number of participants with positive account balances, the age of the plan in years, and dummy variables indicating whether the plan is a 401(k) plan, whether it offers auto enrollment, whether it offers company stock as an investment option, whether it offers any mutual funds as investment options, whether it offers mutual funds, separate accounts, or collective trusts that behave like TDFs, whether it offers mutual fund TDFs, and whether it has a single record keeper (SRK). For the subset of 7,687 plans that offer TDFs and have a single record keeper that is an asset management firm, we calculate the fraction of TDFs and non-TDFs that are managed by the SRK. We report several measures of firm risk. For those firms with publicly-traded equity, we estimate a CAPM beta (using the 24 monthly stock returns through December 2009). In addition, we report the standard deviation of actual monthly returns (over the same 24 months), the standard deviation of predicted monthly returns (based on the CAPM beta and return on the market portfolio), and the standard deviation of the residual monthly returns. To determine the industry-level CAPM beta, we assign each firm the median CAPM beta of the sample of publicly-traded firms that share the same first 3 digits of the North American Industrial Classification System (NAICS). To measure mutual fund risk, we estimate a CAPM beta (using the 24 monthly fund returns through December 2009). We report estimated betas separately for TDFs with target retirement dates of 2010, 2020, 2030, 2040, and 2050, for the full sample of TDFs, and for the sample of non-TDFs. The number of observations varies both because not all plans offer TDFs and because not all mutual funds could be matched to CRSP.

\begin{tabular}{|c|c|c|c|c|c|}
\hline & $\mathrm{N}$ & Mean & Std. Dev. & Min & Max \\
\hline \multicolumn{6}{|l|}{ Plan characteristics in 2010} \\
\hline Assets (in millions) & 16,766 & 134.62 & 708.67 & 0.01 & $36,741.60$ \\
\hline Number of participants (in thousands) & 16,766 & 2.00 & 8.08 & 0.00 & 306.61 \\
\hline Plan age in years & 16,766 & 22.94 & 13.45 & 0.00 & 95.00 \\
\hline 401(k) plan? & 16,766 & 0.91 & 0.29 & 0.00 & 1.00 \\
\hline Auto enrollment? & 16,766 & 0.23 & 0.42 & 0.00 & 1.00 \\
\hline Offers company stock? & 16,766 & 0.13 & 0.33 & 0.00 & 1.00 \\
\hline Offers any mutual funds? & 16,766 & 0.85 & 0.36 & 0.00 & 1.00 \\
\hline Offers any TDFs? & 16,766 & 0.66 & 0.47 & 0.00 & 1.00 \\
\hline Offers mutual fund TDFs? & 16,766 & 0.50 & 0.50 & 0.00 & 1.00 \\
\hline Single record keeper (SRK)? & 16,766 & 0.75 & 0.43 & 0.00 & 1.00 \\
\hline Fraction of TDFs managed by SRK? & 7,687 & 0.73 & 0.44 & 0.00 & 1.00 \\
\hline Fraction of non-TDFs managed by SRK? & 7,687 & 0.38 & 0.27 & 0.00 & 1.00 \\
\hline \multicolumn{6}{|l|}{ Measures of firm risk in 2009} \\
\hline CAPM beta (firm-level) & 1,740 & 1.37 & 0.91 & -1.26 & 8.65 \\
\hline Standard deviation of total returns & 1,740 & 0.17 & 0.10 & 0.04 & 1.27 \\
\hline Standard deviation of predicted returns & 1,740 & 0.10 & 0.06 & 0.00 & 0.60 \\
\hline Standard deviation of residual returns & 1,740 & 0.14 & 0.08 & 0.03 & 1.12 \\
\hline CAPM beta (3-digit industry-level) & 16,301 & 1.21 & 0.48 & 0.14 & 2.57 \\
\hline \multicolumn{6}{|l|}{ Measures of mutual fund risk in 2009} \\
\hline CAPM beta of 2010 TDF & 6,677 & 0.63 & 0.07 & 0.40 & 0.90 \\
\hline CAPM beta of $2020 \mathrm{TDF}$ & 7,581 & 0.78 & 0.06 & 0.63 & 1.00 \\
\hline CAPM beta of 2030 TDF & 7,491 & 0.91 & 0.04 & 0.76 & 1.03 \\
\hline CAPM beta of 2040 TDF & 7,641 & 0.96 & 0.04 & 0.85 & 1.04 \\
\hline CAPM beta of $2050 \mathrm{TDF}$ & 6,504 & 0.98 & 0.04 & 0.87 & 1.04 \\
\hline Average CAPM beta of mutual fund TDFs & 8,277 & 0.79 & 0.06 & 0.32 & 1.02 \\
\hline Average CAPM beta of other mutual funds & 14,064 & 0.83 & 0.15 & -1.69 & 1.58 \\
\hline
\end{tabular}




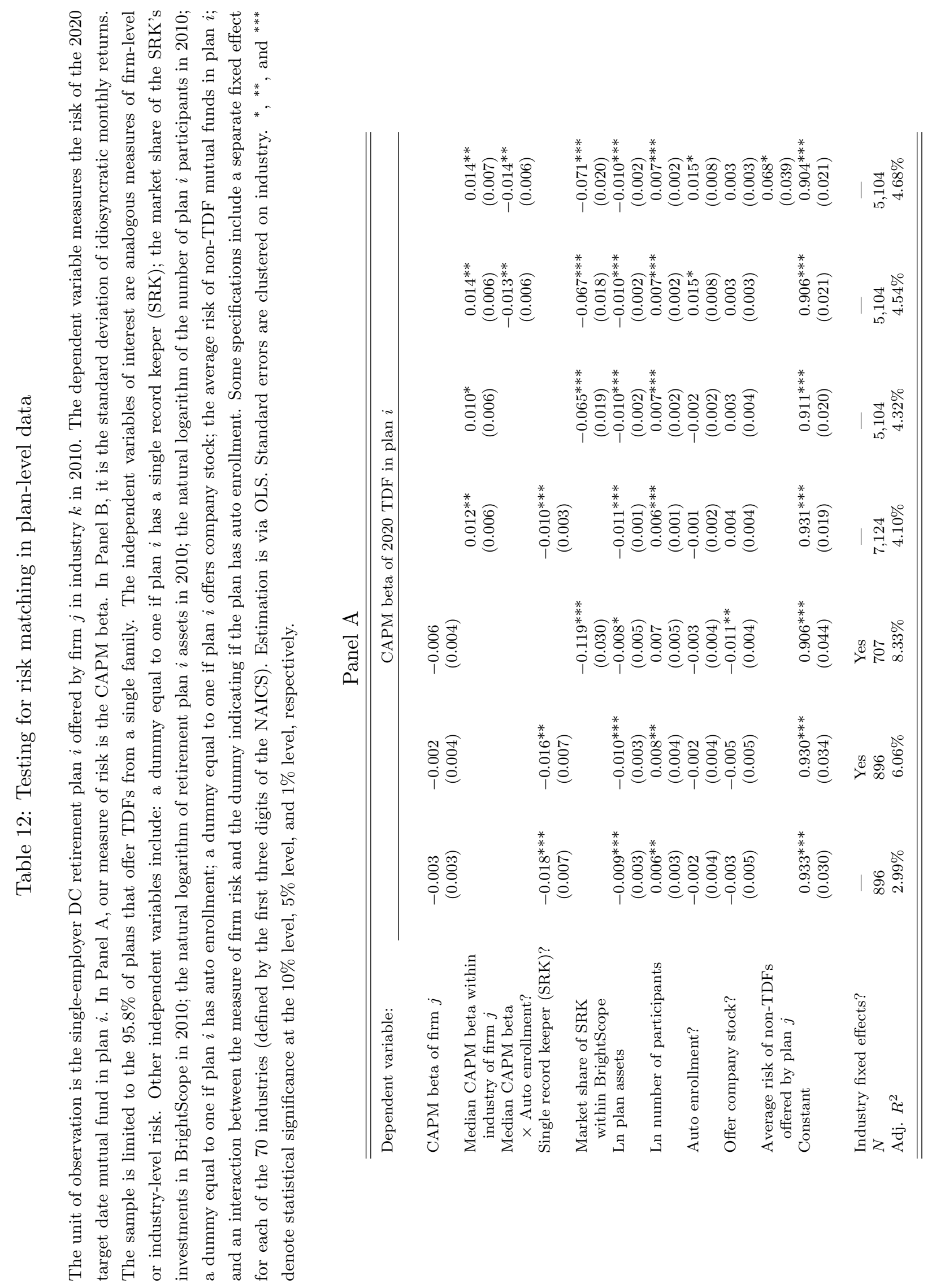




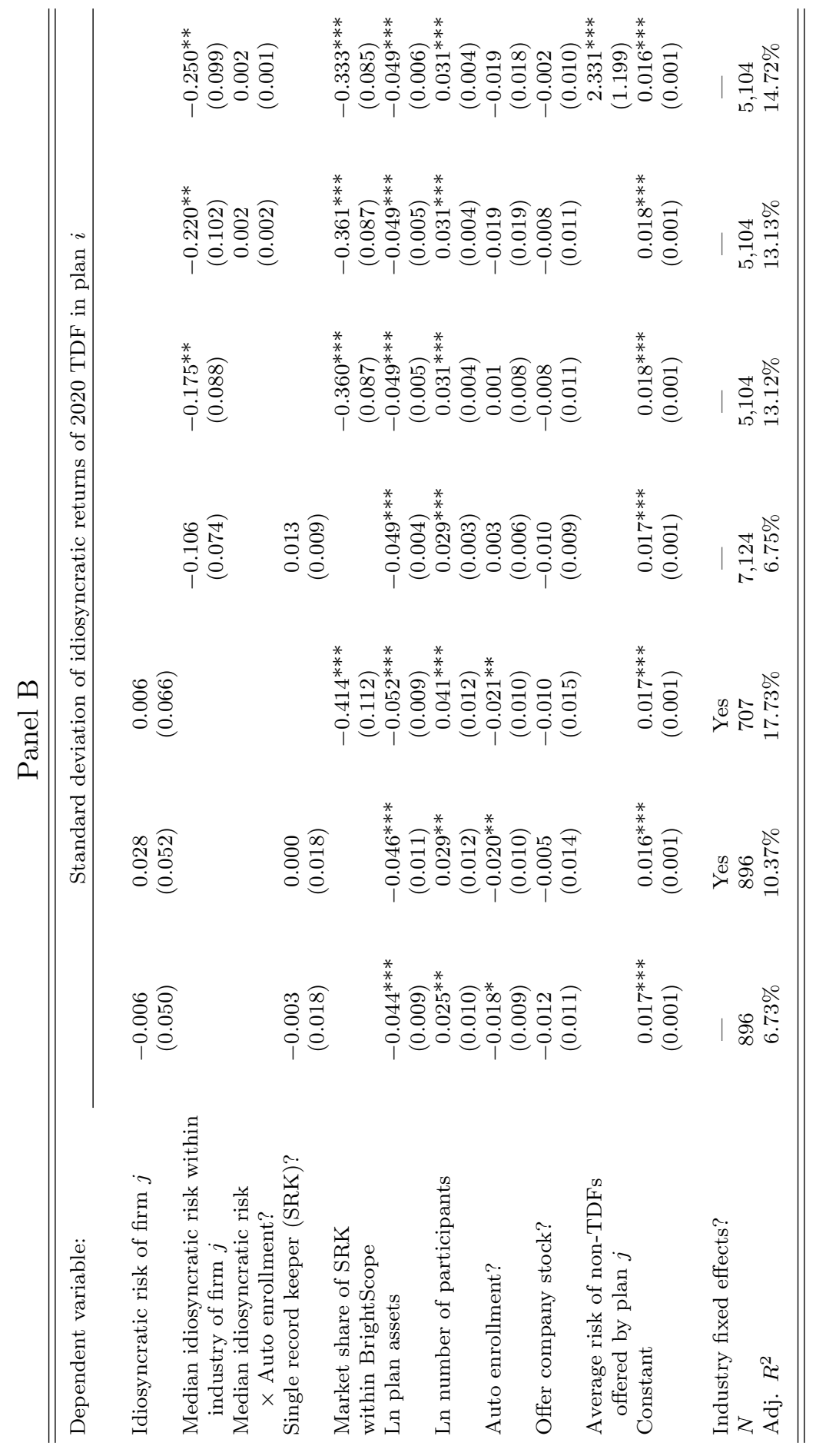




\section{Heterogeneity in Target-Date Funds: Optimal Risk-Taking or Risk Matching? \\ Supplementary Appendix}

June 30, 2015 


\section{A Investor's utility costs}

In this section, we analyze the utility costs that a TDF investor is exposed to when: (i) the TDF equity allocation differs from the optimal allocation; (ii) the TDF equity allocation differs from the optimal allocation and there is uncertainty surrounding the equity premium; (iii) the TDF manager generates idiosyncratic risk, and this idiosyncratic risk is not accounted for in the TDF's asset allocation choice. The analysis is cast in a simple mean-variance setting with investment in the equity index and a risk-free asset. This setting allows us to derive simple analytical closed-form expressions for the utility costs associated with sub-optimal policies. Obviously, the quantitative implications of our analysis only have illustrative value, as our model abstracts from the intertemporal and human capital considerations that are the very motivation for the glide paths offered by TDFs.

\section{A.1 The basic setting}

Assume individual investors have utility defined over the first two moments of yearly portfolio returns, and the investor can invest in equities and the risk-free asset:

$$
U=r_{f}+w \mu-\frac{\gamma}{2} w^{2} \sigma^{2}
$$

where $r_{f}$ is the risk-free rate, $w$ is the equity allocation, $\mu$ is the equity risk premium, and $\sigma$ is equity volatility. Note that the utility function above equals the certainty-equivalent return (CER) of the portfolio.

The optimal equity allocation is:

$$
w^{\star}=\frac{\mu}{\gamma \sigma^{2}} .
$$


Let $x \equiv w-w^{*}$ denote the difference between the actual equity allocation and the optimal allocation. The difference in CERs between the optimal and the actual allocations is a measure of the utility cost: it is (approximately) the percentage of wealth that an investor facing the sub-optimal allocation would be willing to forgo, to be able to implement the optimal allocation instead of the sub-optimal allocation. ${ }^{1}$ We have:

$$
\begin{aligned}
\mathrm{CER}^{\star}-\mathrm{CER} & =\left(w^{\star}-w\right) \mu-\frac{\gamma}{2}\left[\left(w^{\star}\right)^{2}-w^{2}\right] \sigma^{2} \\
& \equiv x \mu+\frac{\gamma}{2}\left(x^{2}-2 x w^{\star}\right) \sigma^{2} \\
& =\frac{\gamma}{2} \sigma^{2} x^{2}+\left(\mu-w^{\star} \gamma \sigma^{2}\right) x \\
& =\frac{\gamma}{2} \sigma^{2} x^{2} .
\end{aligned}
$$

For a given departure from optimality $x$, the utility cost increases with $\gamma$ and $\sigma$. While the Envelope Theorem tells us that departures from optimality do not matter in a neighborhood of $x=0$, as the departures increase in (absolute) magnitude, so do the utility costs, which are increasing in both the risk aversion of the investor and the volatility of equity returns.

The point above is illustrated in Figure 1, where we plot the utility costs of departures from optimality, as a function of the absolute size of the deviation, for different values of $\gamma$, assuming $\sigma=0.205$ - the same value chosen by Gomes et al. (2009). The costs are reported in percentage points and are scaled by 45, the time horizon of an investor who starts saving at 21 and plans to retire at $65 .^{2}$ To get a sense of the magnitudes involved, for a relatively minor departure from optimality of $10 \%$, the utility cost for an investor with $\gamma=8$ is $7.46 \%$.

\footnotetext{
${ }^{1}$ This is the measure of utility costs used, for example, in Balduzzi and Lynch (1999).

${ }^{2}$ This would be the difference in CERs for an investor facing i.i.d. returns, and who has preferences over the mean and variance of $T$-period simple returns:

$$
U=T r_{f}+w(T \mu)-\frac{\gamma}{2} w^{2}\left(T \sigma^{2}\right) .
$$
}




\section{A.2 The effect of parameter uncertainty}

We now assume that the equity premium $\mu$ is not known and that the posterior density of the equity premium has volatility $\sigma_{\mu}$ (for simplicity, we assume that the volatility of equity returns $\sigma$ is known). A Bayesian investor chooses:

$$
w^{\star}=\frac{\mu}{\gamma\left(\sigma^{2}+\sigma_{\mu}^{2}\right)}
$$

where $\sigma^{2}+\sigma_{\mu}^{2}$ is the variance of the predictive density of returns. ${ }^{3}$ So, the uncertainty surrounding the mean estimate reduces the optimal allocation to the risky asset.

When we compute the utility cost of a sub-optimal allocation, we have:

$$
\mathrm{CER}^{\star}-\mathrm{CER}=\frac{\gamma}{2}\left(\sigma^{2}+\sigma_{\mu}^{2}\right) x^{2}
$$

Hence, uncertainty surrounding the equity premium increases the utility cost associated with a given departure from optimality.

\section{A.3 The role of idiosyncratic risk}

Assume that the TDF, by performing security selection, may outperform or underperform the equity index, but adds idiosyncratic risk to the portfolio. There are now two possible sources of utility costs. First, assume that idiosyncratic risk is ignored by the fund manager. Given her preferences, the investor would want the equity allocation:

$$
w_{\alpha}^{\star}=\frac{\mu+\alpha}{\gamma\left(\sigma^{2}+\sigma_{\epsilon}^{2}\right)},
$$

\footnotetext{
${ }^{3}$ This follows from the fact that $\operatorname{Var}(r \mid \mathbf{X}, \sigma, m)=\operatorname{Var}(r \mid \mu)+\operatorname{Var}(\mu \mid \mathbf{X}, \sigma, m)$, where $r$ denotes the equity return, $\mathbf{X}$ denotes a vector of data realizations, and $m$ denotes the mean of the prior density.
} 
where $\alpha$ denotes the expected idiosyncratic return and $\sigma_{\epsilon}$ is the volatility of the idiosyncratic return. Instead, the fund manager ignores $\alpha$ and $\sigma_{\epsilon}$ and selects:

$$
w=\frac{\mu}{\gamma \sigma^{2}}
$$

The utility cost of ignoring the idiosyncratic component of returns is (see equation (3)):

$$
\mathrm{CER}_{\alpha}^{\star}-\mathrm{CER}=\frac{\gamma}{2}\left(\sigma^{2}+\sigma_{\epsilon}^{2}\right)\left(w-w_{\alpha}^{\star}\right)^{2}
$$

As in equation (3), the utility cost is increasing in $\gamma$ and in the total volatility of the returns on the equity allocation.

The second utility costs arises even if the manager optimally accounts for the mean and volatility of idiosyncratic returns. Without idiosyncratic risk, the maximized CER is:

$$
\mathrm{CER}^{\star}=r_{f}+w^{\star} \mu-\frac{\gamma}{2}\left(w^{\star}\right)^{2} \sigma^{2}=r_{f}+\frac{\mu^{2}}{\gamma \sigma^{2}}-\frac{\gamma}{2} \frac{\mu^{2}}{\gamma^{2} \sigma^{4}} \sigma^{2}=r_{f}+\frac{1}{2} \frac{\mu^{2}}{\gamma \sigma^{2}},
$$

where $r_{f}$ denotes the risk-free rate. With idiosyncratic risk, the maximized CER is:

$$
\mathrm{CER}_{\alpha}^{\star}=r_{f}+\frac{1}{2} \frac{(\mu+\alpha)^{2}}{\gamma\left(\sigma^{2}+\sigma_{e}^{2}\right)}
$$

Hence, the utility cost from investing in a TDF that allocates optimally, but has idiosyncratic risk, as opposed to investing in a TDF that allocates optimally, and has no idiosyncratic risk, is:

$$
\mathrm{CER}^{\star}-\mathrm{CER}_{\alpha}^{\star}=\frac{1}{2 \gamma}\left[\frac{\mu^{2}}{\sigma^{2}}-\frac{(\mu+\alpha)^{2}}{\sigma^{2}+\sigma_{e}^{2}}\right]
$$

This cost decreases with $\gamma$, as a higher $\gamma$ reduces the maximized CER, regardless of whether there 
is idiosyncratic risk. This cost increases with $\sigma_{\epsilon}$, as higher idiosyncratic risk reduces the maximized CER in the presence of idiosyncratic risk.

If we sum up the two utility costs,

$$
\mathrm{CER}_{\alpha}^{\star}-\mathrm{CER}+\mathrm{CER}^{\star}-\mathrm{CER}_{\alpha}^{\star}=\mathrm{CER}^{\star}-\mathrm{CER}
$$

we obtain the total utility cost of being invested in a TDF that generates idiosyncratic risk in its equity allocation, but allocates funds ignoring the idiosyncratic risk, relative to a fund that does not generate idiosyncratic risk.

The point above is illustrated in Figure 2, where we use the same assumptions as in Figure 1 , and we set $\alpha=-0.007, \sigma_{\epsilon}=0.01 .^{4}$ In this case, a $10 \%$ deviation from optimality leads to an $11.02 \%$ utility cost.

\footnotetext{
${ }^{4}$ These values are based on the pooled average annual alpha and average annual idiosyncratic volatility in our sample.
} 


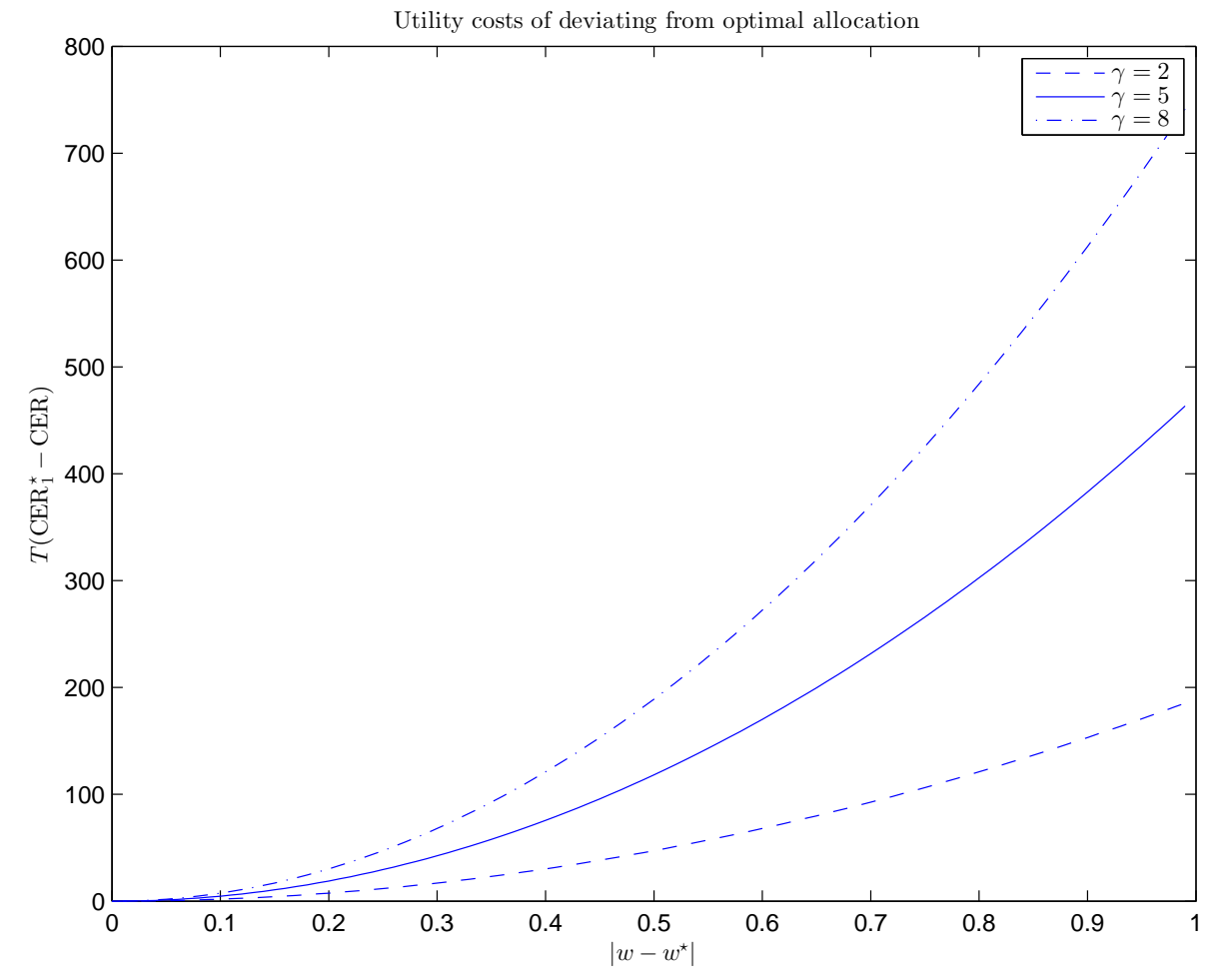

Figure 1: This figure plots $\mathrm{CER}^{\star}-\mathrm{CER}$ as a function of $\left|w^{\star}-w\right|$. 


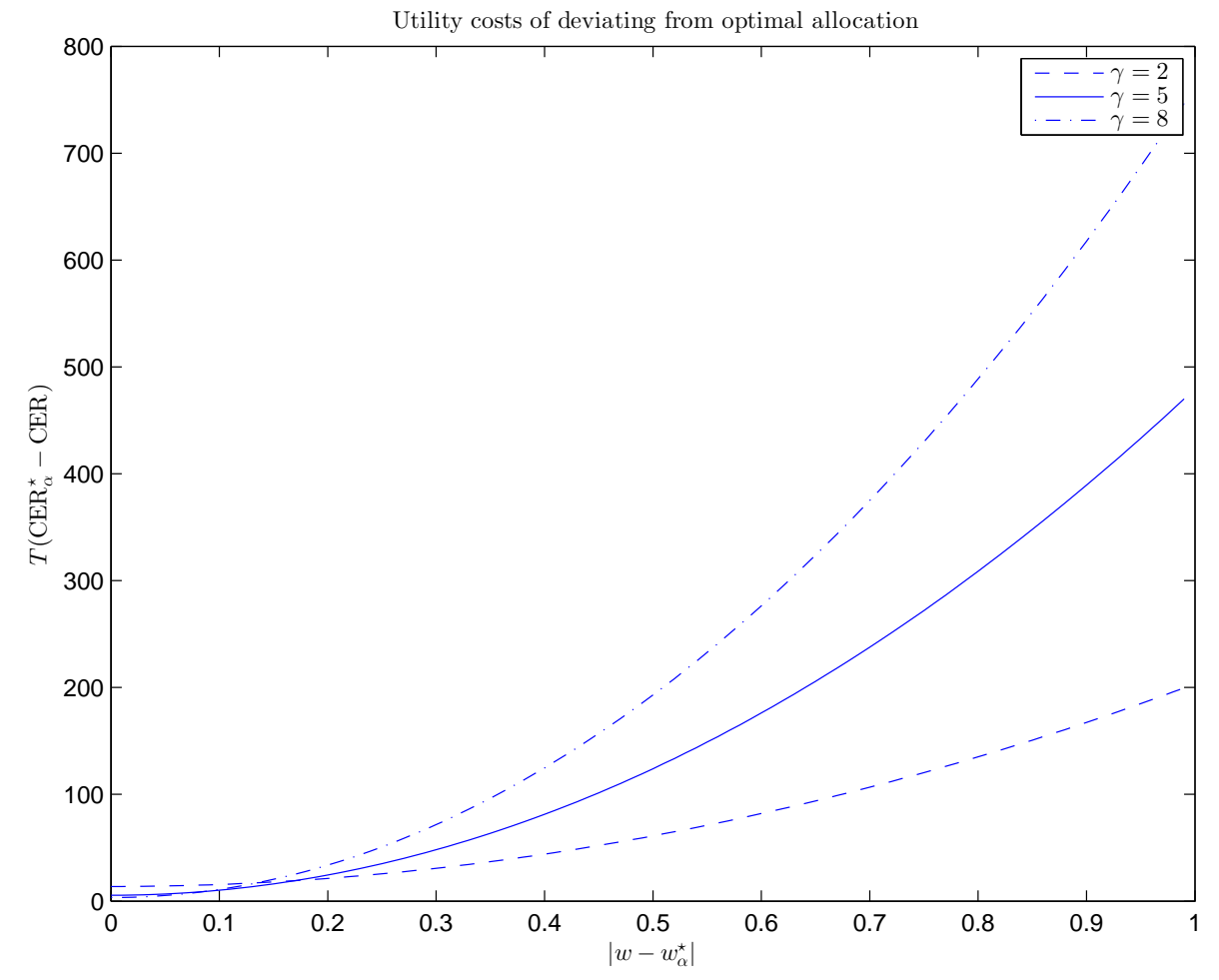

Figure 2: This figure plots $\mathrm{CER}_{\alpha}^{\star}-\mathrm{CER}$ as a function of $\left|w_{\alpha}^{\star}-w\right|$. 


\section{B Inconsistencies in CRSP equity holdings data}

In the earlier versions of this paper, we used CRSP data on allocations to equity, bonds, and cash, to document dispersion in TDF glide paths. However, after downloading a version of the CRSP mutual fund database that extended our sample through 2012, we lost faith in the quality of these CRSP variables. (This is why, in the current version, we test for dispersion in glide paths by testing for dispersion in factor loadings estimated using daily returns.)

This section of the appendix documents significant differences in the fraction of a TDF's portfolio invested in common stock (PER_COM) between the old and new versions of the CRSP data. CRSP changed data vendors, resulting in "new" historical data for PER_COM from 1998 to the present. Table B.1 compares the availability of equity holdings data for 5,870 share class-level observations between 1994 and 2009. We observe either PER_COM_OLD or PER_COM_NEW for $93.3 \%$ of the observations. However, we possess both PER_COM_OLD and PER_COM_NEW for only $77.0 \%$ of the observations. Moreover, the correlation between PER_COM_NEW and PER_COM_OLD is only 0.5608. Because TDFs are structured as funds of funds they disclose their holdings of the underlying funds rather than their indirect holdings of equity and debt. This

likely explains the large number of observations for which PER_COM_NEW or PER_COM_OLD is missing or coded as zero.

Table B.2 calculates the average difference between PER_COM_NEW and PER_COM_OLD for different samples of TDFs. The unit of observations is TDF portfolio $i$ in calendar year $t$. We drop any TDF-year observation for which PER_COM_NEW or PER_COM_OLD equals zero. The average difference is close to zero, but there are significant differences across calendar years $(-11.8 \%$ in 2004 to $16.9 \%$ in 2006), target date differences (6.4\% for 2010 TDFs and $-4.2 \%$ for 2050 TDFs), and target-date-year cells (-24.9\% to $32.0 \%)$. 
Table B.1: Comparing equity holdings in NEW and OLD versions of CRSP Mutual Fund Data

This table compares equity holdings data from two different versions of the CRSP mutual fund database. The OLD version was downloaded from WRDS in 2010 and the NEW version was downloaded in 2013. The sample is limited to TDFs. The unit of observation is share class $i$ in year $t$. We observe equity holdings (PER_COM) from both versions for $77.0 \%$ of the observations. The correlation between PER_COM_NEW and PER_COM_OLD is 0.5608.

\begin{tabular}{|c|c|c|c|c|c|c|c|c|c|c|c|c|}
\hline & \multirow{2}{*}{$\frac{\text { ALL }}{\#}$} & \multicolumn{2}{|c|}{ NEW or OLD } & \multicolumn{3}{|c|}{ NEW and OLD } & \multicolumn{2}{|c|}{ NEW only } & \multicolumn{2}{|c|}{ OLD only } & \multicolumn{2}{|c|}{ Neither } \\
\hline & & \# & $\%$ & \# & $\%$ & Corr. & \# & $\%$ & $\#$ & $\%$ & \# & $\%$ \\
\hline 1994 & 10 & 10 & $100.0 \%$ & 10 & $100.0 \%$ & 1.0000 & 0 & $0.0 \%$ & 0 & $0.0 \%$ & 0 & $0.0 \%$ \\
\hline 1995 & 10 & 10 & $100.0 \%$ & 10 & $100.0 \%$ & 1.0000 & 0 & $0.0 \%$ & 0 & $0.0 \%$ & 0 & $0.0 \%$ \\
\hline 1996 & 15 & 15 & $100.0 \%$ & 15 & $100.0 \%$ & 1.0000 & 0 & $0.0 \%$ & 0 & $0.0 \%$ & 0 & $0.0 \%$ \\
\hline 1997 & 18 & 18 & $100.0 \%$ & 18 & $100.0 \%$ & 1.0000 & 0 & $0.0 \%$ & 0 & $0.0 \%$ & 0 & $0.0 \%$ \\
\hline 1998 & 24 & 19 & $79.2 \%$ & 0 & $0.0 \%$ & & 0 & $0.0 \%$ & 19 & $79.2 \%$ & 5 & $20.8 \%$ \\
\hline 1999 & 35 & 30 & $85.7 \%$ & 0 & $0.0 \%$ & & 0 & $0.0 \%$ & 30 & $85.7 \%$ & 5 & $14.3 \%$ \\
\hline 2000 & 36 & 22 & $61.1 \%$ & 0 & $0.0 \%$ & & 0 & $0.0 \%$ & 22 & $61.1 \%$ & 14 & $38.9 \%$ \\
\hline 2001 & 69 & 31 & $44.9 \%$ & 0 & $0.0 \%$ & & 0 & $0.0 \%$ & 31 & $44.9 \%$ & 38 & $55.1 \%$ \\
\hline 2002 & 87 & 37 & $42.5 \%$ & 6 & $6.9 \%$ & 0.9969 & 0 & $0.0 \%$ & 31 & $35.6 \%$ & 50 & $57.5 \%$ \\
\hline 2003 & 146 & 57 & $39.0 \%$ & 30 & $20.5 \%$ & 0.9097 & 15 & $10.3 \%$ & 12 & $8.2 \%$ & 89 & $61.0 \%$ \\
\hline 2004 & 261 & 134 & $51.3 \%$ & 35 & $13.4 \%$ & 0.5523 & 30 & $11.5 \%$ & 69 & $26.4 \%$ & 127 & $48.7 \%$ \\
\hline 2005 & 460 & 426 & $92.6 \%$ & 208 & $45.2 \%$ & 0.4194 & 104 & $22.6 \%$ & 114 & $24.8 \%$ & 34 & $7.4 \%$ \\
\hline 2006 & 690 & 670 & $97.1 \%$ & 505 & $73.2 \%$ & 0.3880 & 45 & $6.5 \%$ & 120 & $17.4 \%$ & 20 & $2.9 \%$ \\
\hline 2007 & 1,069 & 1,063 & $99.4 \%$ & 846 & $79.1 \%$ & 0.3137 & 34 & $3.2 \%$ & 183 & $17.1 \%$ & 6 & $0.6 \%$ \\
\hline 2008 & 1,476 & 1,472 & $99.7 \%$ & 1,394 & $94.4 \%$ & 0.7862 & 30 & $2.0 \%$ & 48 & $3.3 \%$ & 4 & $0.3 \%$ \\
\hline 2009 & 1,464 & 1,461 & $99.8 \%$ & 1,445 & $98.7 \%$ & 0.7010 & 9 & $0.6 \%$ & 7 & $0.5 \%$ & 3 & $0.2 \%$ \\
\hline ALL & 5,870 & 5,475 & $93.3 \%$ & 4,522 & $77.0 \%$ & 0.5608 & 267 & $4.5 \%$ & 686 & $11.7 \%$ & 395 & $6.7 \%$ \\
\hline
\end{tabular}


Table B.2: Changes in equity holdings from OLD to NEW versions of CRSP Mutual Fund Data This table reports the average difference between PER_COM_NEW and PER_COM_OLD. The unit of observation is portfolio $i$ in year $t$. The sample is limited to TDFs for which we observe both PER_COM_NEW and PER_COM_OLD, and for which neither variable equals zero.

\begin{tabular}{lrrrrrr}
\hline \hline & 2010 & 2020 & 2030 & 2040 & 2050 & ALL \\
\cline { 2 - 4 } \cline { 7 - 7 } 2002 & $-0.19 \%$ & & $-2.24 \%$ & $-1.92 \%$ & & $-1.45 \%$ \\
2003 & $-5.05 \%$ & $-7.18 \%$ & $-6.79 \%$ & $-0.83 \%$ & & $-4.96 \%$ \\
2004 & $-0.80 \%$ & $0.40 \%$ & $-24.94 \%$ & $-21.91 \%$ & & $-11.81 \%$ \\
2005 & $7.80 \%$ & $23.65 \%$ & $5.50 \%$ & $8.35 \%$ & $1.86 \%$ & $10.68 \%$ \\
2006 & $32.00 \%$ & $27.26 \%$ & $12.95 \%$ & $3.12 \%$ & $4.72 \%$ & $16.91 \%$ \\
2007 & $22.69 \%$ & $23.59 \%$ & $11.61 \%$ & $4.82 \%$ & $5.77 \%$ & $13.76 \%$ \\
2008 & $-4.14 \%$ & $-4.40 \%$ & $-5.68 \%$ & $-5.59 \%$ & $-6.99 \%$ & $-5.39 \%$ \\
2009 & $-5.89 \%$ & $-6.36 \%$ & $-6.30 \%$ & $-6.48 \%$ & $-7.25 \%$ & $-6.48 \%$ \\
ALL & $6.39 \%$ & $4.75 \%$ & $-0.07 \%$ & $-2.52 \%$ & $-4.22 \%$ & $0.80 \%$ \\
\hline \hline
\end{tabular}

